JOURNAL OF THE

AMERICAN MATHEMATICAL SOCIETY

Volume 10, Number 3, July 1997, Pages 693-728

S 0894-0347(97)00240-3

\title{
INFINITESIMAL 1-PARAMETER SUBGROUPS AND COHOMOLOGY
}

\author{
ANDREI SUSLIN, ERIC M. FRIEDLANDER, AND CHRISTOPHER P. BENDEL
}

This is the first of two papers in which we determine the spectrum of the cohomology algebra of infinitesimal group schemes over a field $k$ of characteristic $p>0$. Whereas $[\mathrm{SFB}]$ is concerned with detection of cohomology classes, the present paper introduces the graded algebra $k\left[V_{r}(G)\right]$ of functions on the scheme of infinitesimal 1-parameter subgroups of height $\leq r$ on an affine group scheme $G$ and demonstrates that this algebra is essentially a retract of $H^{e v}(G, k)$ provided that $G$ is an infinitesimal group scheme of height $\leq r$.

This work is a continuation of $[\mathrm{F}-\mathrm{S}]$ in which the existence of certain universal extension classes was established, thereby enabling the proof of finite generation of $H^{*}(G, k)$ for any finite group scheme $G$ over $k$. The role of the scheme of infinitesimal 1-parameter subgroups of $G$ was foreshadowed in $[\mathrm{F}-\mathrm{P}]$ where $H^{*}\left(G_{(1)}, k\right)$ was shown to be isomorphic to the coordinate algebra of the scheme of $p$-nilpotent elements of $g=\operatorname{Lie}(G)$ for $G$ a smooth reductive group, $G_{(1)}$ the first Frobenius kernel of $G$, and $p=\operatorname{char}(k)$ sufficiently large. Indeed, $p$-nilpotent elements of $g$ correspond precisely to infinitesimal 1-parameter subgroups of $G_{(1)}$. Much of our effort in this present paper involves the analysis of the restriction of the universal extension classes to infinitesimal 1-parameter subgroups.

In $\S 1$, we construct the affine scheme $V_{r}(G)$ of homomorphisms from $\mathbb{G}_{a(r)}$ to $G$ which we call the scheme of infinitesimal 1-parameter subgroups of height $\leq r$ in $G$. In the special case $r=1$, this is the scheme of $p$-nilpotent elements of the $p$-restricted Lie algebra $\operatorname{Lie}(G)$; for various classical groups $G, V_{r}(G)$ is the scheme of $r$-tuples of $p$-nilpotent, pairwise commuting elements of Lie $(G)$. More generally, an embedding $G \subset G L_{n}$ determines a closed embedding of $V_{r}(G)$ into the scheme of $r$-tuples of $p$-nilpotent, pairwise commuting elements of $g l_{n}=\operatorname{Lie}\left(G L_{n}\right)$. The relationship between $k\left[V_{r}(G)\right]$, the coordinate algebra of $V_{r}(G)$, and $H^{*}(G, k)$ is introduced in Theorem 1.14: a natural homomorphism of graded $k$-algebras

$$
\psi: H^{e v}(G, k) \rightarrow k\left[V_{r}(G)\right]
$$

is constructed, a map which we show in $[\mathrm{SFB}]$ to induce a bijective map on associated schemes.

The universal classes $e_{r} \in H^{2 p^{r-1}}\left(G L_{n}, g l_{n}^{(r)}\right)$ constructed in [F-S] determine characteristic classes $e_{r}(G, V)$ for any affine group scheme $G$ provided with a finite dimensional representation $V$. Our central computation is the determination in

Received by the editors October 25, 1996 and, in revised form, March 20, 1997.

1991 Mathematics Subject Classification. Primary 14L15, 20G10, 20C20; Secondary 17 B50.

Key words and phrases. Infinitesimal group schemes, 1-parameter subgroups.

The first author was partially supported by I.S.F. grant 0100 and N.S.F. grant DMS - 9510242.

The second author was partially supported by the N.S.F. and the Humboldt Foundation. 
Theorem 4.9 of the characteristic classes associated to 1-parameter subgroups of $G L_{n}$. Since we apply this computation to the universal 1-parameter subgroup

$$
\exp _{\underline{\alpha}}: \mathbb{G}_{a(r)} \otimes_{\mathbb{F}_{p}} A \rightarrow G L_{n(r)} \otimes_{\mathbb{F}_{p}} A,
$$

where $A$ is the coordinate algebra of $V_{r}\left(G L_{n}\right)$, we consider characteristic classes of group schemes over a commutative (unital) ring. §2 presents a (straight-forward) extension of the theory of strict polynomial functors (used in [F-S] to construct the universal classes $e_{r}$ ) from the context of vector spaces over a field to projective modules over a commutative ring. In particular, we formulate the concept and basic properties of base change of such functors.

Since a homomorphism $\exp _{\underline{\alpha}}: \mathbb{G}_{a(r)} \otimes_{\mathbb{F}_{p}} A \rightarrow G L_{n} \otimes_{\mathbb{F}_{p}} A$ specified by the $r$ tuple $\underline{\alpha}=\left(\alpha_{0}, \ldots, \alpha_{r-1}\right)$ is a product of homomorphisms $\exp _{\alpha_{i}} \circ F^{i}: \mathbb{G}_{a(r)} \otimes_{\mathbb{F}_{p}}$ $A \rightarrow \mathbb{G}_{a(r)} \otimes_{\mathbb{F}_{p}} A \rightarrow G L_{n} \otimes_{\mathbb{F}_{p}} A$ (where $F$ denotes the Frobenius map), we find it useful to introduce a coproduct structure on certain Ext-groups in the category of strict polynomial functors. This is done in $\S 3$ by investigating Ext-algebras in the category $\mathcal{P}(2)$ of strict polynomial bifunctors. The complete determination of the Hopf algebra $\operatorname{Ext}_{\mathcal{P}}^{*}\left(I^{(r)}, I^{(r)}\right)$ is achieved in $\S 4$.

The universal classes $e_{i}^{(r-i)}, 1 \leq i \leq r$, determine a natural map

$$
\phi: S^{*}\left(\bigoplus_{1 \leq i \leq r} g l_{n}^{(r) \#}\left[2 p^{i-1}\right]\right) \rightarrow H^{e v}\left(G L_{n(r)}, k\right)
$$

where the symmetric algebra can be viewed as the algebra of regular functions (suitably graded with appropriate Frobenius twists) on the affine scheme $g l_{n}^{(r) \times r}$. The explicit form of the characteristic classes $e_{r}(j)\left(\left(\mathbb{G}_{a(r)}\right)_{A}, V_{\underline{\alpha}}\right)$ given in Theorem 4.9 enables us to verify a very pleasing description of $\psi \circ \phi$. Indeed, Theorem 5.2 asserts that the composition of the maps of schemes

$$
V_{r}(G) \stackrel{\Psi}{\longrightarrow} \operatorname{Spec}\left(H^{e v}(G, k)\right) \stackrel{\Phi}{\longrightarrow} g l_{n}^{(r) \times r}
$$

determined by $\phi$ and $\psi$ is merely the $r$-th Frobenius twist of the natural embedding $V_{r}(G) \subset g l_{n}^{\times r}$.

Finally, $\S 6$ determines the homology algebra of the infinite general linear group over a finite field with coefficients in the adjoint representation. This multiplicative structure is easily deduced from our determination of the coproduct structure introduced in $\S 3$ together with computations of [F-S].

The second author gratefully acknowledges the hospitality of the University of Heidelberg during the preparation of this paper.

\section{$\S 1$. The scheme $V_{r}(G)$}

As we establish in this section, the set of 1-parameter subgroups $\mathbb{G}_{a(r)} \rightarrow G$ of height $r$ of an affine group scheme $G$ admits the structure of the set of rational points of an affine $k$-scheme $V_{r}(G)$ whose coordinate algebra has the natural structure of a graded, connected $k$-algebra. Moreover, whenever $G=G L_{n}$ or a classical group, then $V_{r}(G)$ can be identified as the scheme of $r$-tuples of $p$-nilpotent, pairwise commuting elements of $g=\operatorname{Lie}(G)$. A connection between the scheme $V_{r}(G)$ and the cohomology of $G$ is established in Theorem 1.14.

The following definition was first formulated by D. Gross [G]. As usual, $k$ will denote a field of characteristic $p>0$. 
Definition 1.1. Let $G$ be an affine group scheme/ $k$ and $r>0$ be a positive integer. We define the functor

$$
V_{r}(G):(\operatorname{comm} k \text {-alg }) \rightarrow(\text { sets })
$$

by setting

$$
V_{r}(G)(A)=H_{o m} r / A\left(\mathbb{G}_{a(r)} \otimes_{k} A, G \otimes_{k} A\right) .
$$

Proposition 1.2. For any commutative $k$-algebra $A$, we have a natural identification of $V_{r}\left(G L_{n}\right)(A)$ with

$$
\left\{\left(\alpha_{0}, \ldots, \alpha_{r-1}\right): \alpha_{i} \in M_{n}(A), \alpha_{i}^{p}=0=\left[\alpha_{j}, \alpha_{\ell}\right] \text { for all } 0 \leq i, j, \ell<r\right\} .
$$

Proof. A homomorphism of group schemes over $A$,

$$
f: G_{A} \rightarrow G L_{n} \otimes_{k} A,
$$

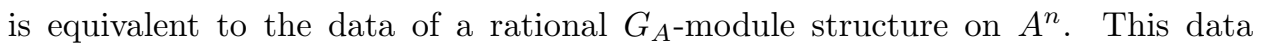
consists of an $A$-module homomorphism

$$
\Delta_{f}: A^{n} \rightarrow A[G] \otimes_{A} A^{n}
$$

making commutative the following two diagrams:

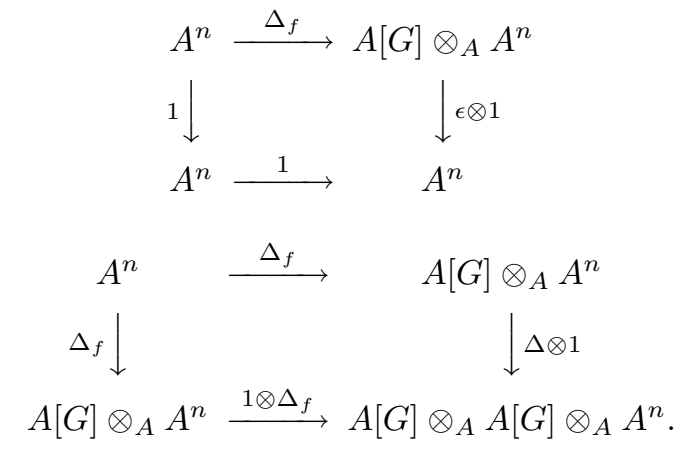

Let $G$ denote $\mathbb{G}_{a(r)}$, so that $A[G]=A[T] / T^{p^{r}}$ is a free $A$-module of rank $p^{r}$. To give the $A$-module homomorphism $\Delta_{f}: A^{n} \rightarrow A[G] \otimes_{A} A^{n}$ defining the rational $G_{A^{-}}$ module structure on $A^{n}$ is equivalent to giving a sequence $\beta_{0}, \ldots, \beta_{p^{r}-1} \in M_{n}(A)$ :

$$
\Delta_{f}(v)=\sum_{i=0}^{p^{r}-1} t^{i} \otimes \beta_{i}(v) .
$$

The commutativity of (1.2.1) is equivalent to the condition that $\beta_{0}=1$. The commutativity of (1.2.2) is equivalent to the conditions

$$
\begin{gathered}
\beta_{i} \beta_{j}=\left(\begin{array}{c}
i+j \\
j
\end{array}\right) \beta_{i+j}, \quad i+j<p^{r}, \\
\beta_{i} \beta_{j}=0, \quad i+j \geq p^{r} .
\end{gathered}
$$

In particular, $\beta_{i}^{p}=0=\left[\beta_{j}, \beta_{\ell}\right]$ for all $0 \leq i, j, \ell<p^{r}$.

Set $\alpha_{i}=\beta_{p^{i}}$. For any $j=\sum_{\ell=0}^{r-1} j_{\ell} p^{\ell}$ with $0 \leq j_{\ell}<p$, the equality

$$
\beta_{j}=\frac{\alpha_{0}^{j_{0}} \cdots \alpha_{r-1}^{j_{r-1}}}{\left(j_{0} !\right) \cdots\left(j_{r-1} !\right)}
$$


can be easily established using induction and the observation that

$$
\left(\begin{array}{c}
j+j^{\prime} \\
j
\end{array}\right) \equiv\left(\begin{array}{c}
j_{0}+j_{0}^{\prime} \\
j_{0}
\end{array}\right) \cdots\left(\begin{array}{c}
j_{r-1}+j_{r-1}^{\prime} \\
j_{r-1}
\end{array}\right) \bmod (p)
$$

(where $j^{\prime}$ similarly equals $\sum j_{\ell}^{\prime} p^{\ell}$ with $0 \leq j_{\ell}^{\prime}<p$ ).

Conversely, given an arbitrary $r$-tuple $\underline{\alpha}=\left(\alpha_{0}, \ldots, \alpha_{r-1}\right) \in M_{n}(A)^{r}$ of commuting $p$-nilpotent matrices, we define $\Delta_{f}$ using $(1.2 .3)$ by defining $\beta_{j}$ according to $(1.2 .5)$. A direct verification shows that $\Delta_{f}$ so defined fits in the commutative squares (1.2.1) and (1.2.2).

Remark 1.3. The same argument as above may be used to show that for any commutative $k$-algebra $A$ we have a natural identification (cf. [D-G])

$$
\begin{aligned}
& \operatorname{Hom}_{G r / A}\left(\mathbb{G}_{a} \otimes_{k} A, G L_{n} \otimes_{k} A\right)=\left\{\left(\alpha_{0}, \alpha_{1}, \ldots\right): \alpha_{i} \in M_{n}(A),\right. \\
& \left.\qquad \alpha_{i}^{p}=0,\left[\alpha_{i}, \alpha_{j}\right]=0 \text { for all } i, j ; \alpha_{i}=0 \text { for } i \text { big enough }\right\} .
\end{aligned}
$$

The homomorphism $\exp _{\underline{\alpha}}: \mathbb{G}_{a} \otimes_{k} A \rightarrow G L_{n} \otimes_{k} A$ corresponding to a sequence $\underline{\alpha}=\left(\alpha_{0}, \alpha_{1}, \ldots\right)$ of pairwise commuting $p$-nilpotent matrices is given by the formula (in which we assume that $\alpha_{i}=0$ for $i \geq r$ )

$$
\exp _{\underline{\alpha}}(s)=\exp \left(s \alpha_{0}\right) \cdot \exp \left(s^{p} \alpha_{1}\right) \cdot \ldots \cdot \exp \left(s^{p^{r-1}} \alpha_{r-1}\right) \in G L_{n}(S)
$$

for any $A$-algebra $S$ and any $s \in S$, where for any $p$-nilpotent matrix $\alpha \in M_{n}(S)$ we set

$$
\exp (\alpha)=1+\alpha+\frac{\alpha^{2}}{2}+\cdots+\frac{\alpha^{p-1}}{(p-1) !} \in G L_{n}(S) .
$$

Whenever $f: \mathbb{G}_{a} \otimes_{k} A \rightarrow G \otimes_{k} A$ is a group homomorphism (over $A$ ), we shall use the notation $f^{(1)}$ for the composition homomorphism $f \circ F$ (where $F$ denotes the Frobenius endomorphism of $\mathbb{G}_{a}$ ). With this notation the formula (1.3.1) may be rewritten as

$$
\exp _{\underline{\alpha}}=\exp _{\alpha_{0}} \cdot \exp _{\alpha_{1}}^{(1)} \cdot \ldots \cdot \exp _{\alpha_{r-1}}^{(r-1)} .
$$

Note finally that the homomorphism $\exp _{\underline{\alpha}}: \mathbb{G}_{a(r)} \otimes_{k} A \rightarrow G L_{n} \otimes_{k} A$ defined by an $r$ tuple of pairwise commuting $p$-nilpotent matrices $\underline{\alpha}=\left(\alpha_{0}, \ldots, \alpha_{r-1}\right)$ coincides with the restriction of the corresponding homomorphism $\exp _{(\underline{\alpha}, 0)}: \mathbb{G}_{a} \otimes_{k} A \rightarrow G L_{n} \otimes_{k} A$ to $\mathbb{G}_{a(r)} \otimes_{k} A$.

As was noted in the proof of Proposition 1.2, to give a homomorphism $\mathbb{G}_{a(r)} \rightarrow$ $G L_{n}$ is the same as to make $k^{n}$ into a comodule over the coalgebra $k\left[\mathbb{G}_{a(r)}\right]$ and hence is the same as to make $k^{n}$ into a module over the algebra $k\left[\mathbb{G}_{a(r)}\right]^{\#}$. The following statement makes explicit this algebra.

Corollary 1.4. Let $v_{0}, \ldots, v_{p^{r}-1}$ be the basis of $k\left[\mathbb{G}_{a(r)}\right]^{\#}=\left(k[T] / T^{p^{r}}\right)^{\#}$ dual to the standard basis of $k[T] / T^{p^{r}}$. Denote $v_{p^{i}}$ by $u_{i}$. Then the algebra $k\left[\mathbb{G}_{a(r)}\right]^{\#}$ coincides with $k\left[u_{0}, \ldots, u_{r-1}\right] /\left(u_{0}^{p}, \ldots, u_{r-1}^{p}\right)$. Moreover for any $0 \leq j \leq p^{r}-1$ we have the following formula (in which $j_{0}, \ldots, j_{r-1}$ are the $p$-adic digits of $j$, i.e. $0 \leq j_{l}<p$ and $\left.j=\sum_{l=0}^{r-1} j_{l} p^{l}\right)$ :

$$
v_{j}=\frac{u_{0}^{j_{0}} \cdot \ldots \cdot u_{r-1}^{j_{r-1}}}{j_{0} ! \cdot \ldots \cdot j_{r-1} !} .
$$

As we see in the proof of the following theorem, the representability of $V_{r}(G)$ is an easy consequence of Proposition 1.2. 
Theorem 1.5. For any affine group scheme $G / k$, the functor $V_{r}(G)$ is represented by an affine scheme of finite type over $k$, which we'll still denote $V_{r}(G)$. Moreover $G \mapsto V_{r}(G)$ is a covariant functor from the category of affine group schemes/ $k$ to the category of affine schemes of finite type/ $k$, which takes closed embeddings (of group schemes) to closed embeddings.

Proof. The equations $\alpha_{i}^{p}=0=\left[\alpha_{j}, \alpha_{\ell}\right], 0 \leq i, j, \ell<p^{r}$, define the affine subscheme of $\mathbb{A}^{r n^{2}}$ which clearly represents the functor $V_{r}\left(G L_{n}\right)$. For an arbitrary $G$ choose an embedding $G \hookrightarrow G L_{n}$ and denote by $F_{1}, \ldots, F_{M} \in k\left[G L_{n}\right]$ the defining equations of $G$. Then $V_{r}(G)$ is given by the additional $M p^{r}$ equations in the " $r n^{2}$ variables" of $\underline{\alpha} \in \mathbb{A}^{r n^{2}}$

$$
F_{i}\left(\exp _{\underline{\alpha}}(t)\right)=0 \in k\left[\mathbb{G}_{a(r)}\right]=k[t]=k[T] / T^{p^{r}} .
$$

Whenever $V / k$ is a finite-dimensional vector space, we shall use the same notation $V$ for the corresponding linear scheme $\operatorname{Spec}\left(S^{*}\left(V^{\#}\right)\right)$ over $k$. Let $g / k$ be a finite dimensional $p$-restricted Lie algebra. The $p$-th power map $[p]: g \rightarrow g$ is homogeneous polynomial (of degree $p$ ) and hence may be considered as a morphism of schemes. We shall denote by $N_{p}(g)$ the $p$-nilpotent cone in $g$, i.e. the fiber over the point $0 \in g$ of the morphism $[p]: g \rightarrow g$, so that for any $k$-algebra $A$ the set of $A$-points of $N_{p}(g)$ may be identified with $\left\{x \in g \otimes_{k} A: x^{[p]}=0\right\}$.

Lemma 1.6. Let $G / k$ be an affine group scheme over $k$ and let $g$ be the corresponding restricted Lie algebra. Then we have a natural identification $V_{1}(G)=N_{p}(g)$.

Proof. Let $A$ be a $k$-algebra. To give a homomorphism $\mathbb{G}_{a(1)} \otimes_{k} A \rightarrow G \otimes_{k} A$ of group schemes over $A$ is the same as to give a homomorphism $\mathbb{G}_{a(1)} \otimes_{k} A \rightarrow G_{(1)} \otimes_{k} A$ (over $A$ ). Since the category of height 1 group schemes is equivalent to the category of $p$-restricted Lie algebras (cf. [J], I.8.5,9.6), this is equivalent to the data of a homomorphism of restricted Lie algebras $g_{a} \otimes_{k} A \rightarrow g \otimes_{k} A$ (over $A$ ), i.e. equivalent to the data of $x \in g \otimes_{k} A$ with $x^{[p]}=0$.

Since we shall demonstrate in $[\mathrm{SFB}]$ that the spectrum of the cohomology algebra $H^{*}(G, k)$ is homeomorphic to $V_{r}(G)$ whenever $G$ is an infinitesimal group scheme of height $\leq r$, it is of interest to obtain as explicit a description of $V_{r}(G)$ as possible. As seen in Lemma 1.8, $V_{r}(G)=V_{r}\left(G_{(r)}\right)$ has a description as $r$-tuples of $p$-nilpotent, pairwise commuting elements of $\operatorname{Lie}(G)$ for classical groups.

Let $G / k$ be an affine group scheme with Lie algebra $g$ and let $\phi: G \hookrightarrow G L_{n}$ be a closed embedding with $d \phi: g \rightarrow g l_{n}$ the associated map of $p$-restricted Lie algebras. We say that $\phi$ is an embedding of exponential type (and $\phi(G) \subset G L_{n}$ is a subgroup of exponential type) if the following equivalent conditions are satisfied:

(1) For every $k$-algebra $A$ and every $p$-nilpotent $x \in g \otimes_{k} A$, the homomorphism $\exp _{d \phi(x)}: \mathbb{G}_{a} \otimes_{k} A \rightarrow G L_{n} \otimes_{k} A$ factors via $G \otimes_{k} A$.

(2) The canonical morphism of schemes

$$
N_{p}(g) \times_{k} \mathbb{G}_{a} \rightarrow N_{p}\left(g l_{n}\right) \times_{k} \mathbb{G}_{a} \stackrel{\exp }{\longrightarrow} G L_{n}
$$

factors via $G$. 
Lemma 1.7. Let $G$ be an affine group scheme with an embedding of exponential type $G \hookrightarrow G L_{n}$. Then

$$
V_{r}(G)(A)=\left\{\underline{\alpha} \in\left(\operatorname{Lie}(G) \otimes_{k} A\right)^{r}: \alpha_{i}^{[p]}=0=\left[\alpha_{j}, \alpha_{l}\right] \text { for all } 0 \leq i, j, l<r\right\} .
$$

Proof. Let $\underline{\alpha}$ be an $r$-tuple in the right hand side of (1.7.1). By assumption, each $\exp _{\alpha_{i}}: \mathbb{G}_{a(r)} \otimes_{k} A \rightarrow G L_{n} \otimes_{k} A$ factors via $G \otimes_{k} A$ and hence so does each $\exp _{\alpha_{i}}^{(i)}$. Thus, the product $\exp _{\underline{\alpha}}$ does as well and so $\exp _{\underline{\alpha}} \in V_{r}(G)$.

Conversely, assume that $\exp _{\underline{\alpha}}: \mathbb{G}_{a(r)} \otimes_{k} A \rightarrow G L_{n} \otimes_{k} A$ factors via $G \otimes_{k} A$. This implies immediately that $\alpha_{0}=d\left(\exp _{\underline{\alpha}}\right)(1) \in\left(\operatorname{Lie}(G) \otimes_{k} A\right) \cap N_{p}\left(g l_{n}\right)(A)=$ $N_{p}(\operatorname{Lie}(G))(A)$. The hypothesis of an embedding of exponential type implies that $\exp _{\alpha_{0}}: \mathbb{G}_{a(r)} \otimes_{k} A \rightarrow G L_{n} \otimes_{k} A$ factors via $G \otimes_{k} A$ and hence $\left(\exp _{\alpha_{1}} \cdot \ldots \cdot \exp _{\alpha_{r-1}}^{(r-2)}\right)^{(1)}$ does so as well. From this we conclude easily that $\exp _{\left(\alpha_{1}, \ldots, \alpha_{r-1}\right)}=\exp _{\alpha_{1}} \cdot \ldots$. $\exp _{\alpha_{r-1}}^{(r-2)}: \mathbb{G}_{a(r-1)} \otimes_{k} A \rightarrow G L_{n} \otimes_{k} A$ factors via $G \otimes_{k} A$ so that induction on $r$ concludes the proof.

The following lemma asserts that various classical subgroups of $G L_{n}$ are of exponential type and hence satisfy the conclusion (1.7.1). We omit the proof which follows by inspection.

Lemma 1.8. (1) Any intersection of subgroups of exponential type of $G L_{n}$ is again of exponential type.

(2) The subgroups $S L_{n} \subset G L_{n}, S p_{2 n} \subset G L_{2 n}, B_{n} \subset G L_{n}$ (subgroup of upper triangular matrices), and $U_{n} \subset G L_{n}$ (subgroup of strictly upper triangular matrices) are of exponential type.

(3) Let $\phi$ be a nondegenerate symmetric bilinear form on the vector space $k^{n}$. Then the orthogonal group $O(\phi) \subset G L_{n}$ is a subgroup of exponential type.

Remark 1.9. Let $G$ be an affine group scheme which admits an embedding $G \hookrightarrow$ $G L_{n}$ of exponential type. The choice of such an embedding gives us an identification of $V_{r}(G)$ as above. We do not know whether this identification is intrinsic or depends upon the choice of an embedding of exponential type.

On the other hand, there are many embeddings which are not of exponential type. For example, if $G$ admits any monomorphism $\mathbb{G}_{a(2)} \rightarrow G$ and if $G \rightarrow G L_{n}$ is an embedding of expontial type, then the composition of this embedding with the embedding $1 \times F: G L_{n} \rightarrow G L_{n} \times G L_{n} \subset G L_{2 n}$ is not of exponential type, where $F$ denotes the Frobenius homomorphism.

We next show that the scheme $V_{r}(G)$ is a cone or, what amounts to the same thing, that the coordinate algebra $k\left[V_{r}(G)\right]$ is graded connected. To do this, we first identify the scheme $V_{r}\left(\mathbb{G}_{a(r)}\right)$.

Lemma 1.10. For any $r>0, V_{r}\left(\mathbb{G}_{a}\right)=V_{r}\left(\mathbb{G}_{a(r)}\right)=\mathbb{A}^{r}$.

Proof. A homomorphism $\mathbb{G}_{a(r)} \otimes_{k} A \rightarrow \mathbb{G}_{a(r)} \otimes_{k} A$ is equivalent to a polynomial $P(T) \in A[T]$ of degree $<p^{r}$ such that $P\left(T+T^{\prime}\right)=P(T)+P\left(T^{\prime}\right)$. Such polynomials are those of the form $P(T)=a_{0} T+a_{1} T^{p}+\ldots+a_{r-1} T^{p^{r-1}}$.

Composition of morphisms defines for each affine group scheme $G$ a natural morphism of schemes

$$
V_{r}(G) \times V_{r}\left(\mathbb{G}_{a(r)}\right) \rightarrow V_{r}(G)
$$


Taking $G=\mathbb{G}_{a(r)}$ we see that $V_{r}\left(\mathbb{G}_{a(r)}\right)$ has a natural structure of a monoid scheme over $k$. Moreover for an arbitrary group scheme $G$ the above morphism defines a right monoid action of $V_{r}\left(\mathbb{G}_{a(r)}\right)$ on $V_{r}(G)$. The monoid structure on $V_{r}\left(\mathbb{G}_{a(r)}\right)$ corresponds to the composition of additive polynomials and so is relatively complicated. To simplify matters we restrict our attention to an abelian submonoid $\mathbb{A}^{1} \subset \mathbb{A}^{r}$, corresponding to linear polynomials. The monoid structure on this monoid $\mathbb{A}^{1}$ is just the usual multiplication.

Lemma 1.11. A right monoid action of $\mathbb{A}^{1}$ on an affine scheme $X=S p e c A$ over $k$ is equivalent to the data of a (nonnegative) grading on the ring $A$.

Proof. For any graded $k$-algebra $A$ we have a canonical $k$-algebra homomorphism

$$
A \rightarrow A[T]: a=a_{0}+a_{1}+\ldots \mapsto a_{0}+a_{1} T+a_{2} T^{2}+\ldots
$$

which defines a morphism of schemes over $k: X \times \mathbb{A}^{1}=$ Spec $A[T] \rightarrow$ Spec $A=X$. One readily verifies that this defines a monoid action of $\mathbb{A}^{1}$ on $X$.

Conversely, assume that we are given a right monoid action of $\mathbb{A}^{1}$ on $X$ :

$$
f: X \times \mathbb{A}^{1} \rightarrow X \text {. }
$$

Consider the corresponding homomorphism of $k$-algebras $f^{*}: A=k[X] \rightarrow$ $k\left[X \times \mathbb{A}^{1}\right]=A[T]$ and set $A_{i}=\left\{a \in A: f^{*}(a) \in A \cdot T^{i}\right\}$. It's easy to check that this defines a nonnegative grading on $A$ and moreover the above two constructions are mutually inverse.

We sum up the results of the above discussion in the following lemma.

Lemma 1.12. For any affine group scheme $G / k$, the coordinate algebra $k\left[V_{r}(G)\right]$ is a graded connected $k$-algebra. Moreover the homomorphism $k\left[V_{r}\left(G^{\prime}\right)\right] \rightarrow k\left[V_{r}(G)\right]$ corresponding to a group scheme homomorphism $G \rightarrow G^{\prime}$ respects gradings.

Proof. The only thing left to prove is the connectedness of $k\left[V_{r}(G)\right]$. Consider first the case $G=G L_{n}$. In this case it's straightforward to check that the canonical action

$$
V_{r}\left(G L_{n}\right) \times \mathbb{A}^{1} \rightarrow V_{r}\left(G L_{n}\right)
$$

corresponds to the following map of $A$-valued points (for any $k$-algebra $A$ ):

$$
\left(\alpha_{0}, \alpha_{1}, \ldots, \alpha_{r-1}\right) \times a \mapsto\left(a \alpha_{0}, a^{p} \alpha_{1}, \ldots, a^{p^{r-1}} \alpha_{r-1}\right) .
$$

The closed embedding $V_{r}\left(G L_{n}\right) \hookrightarrow\left(\mathbb{A}^{n^{2}}\right)^{r}$ defines a surjective $k$-algebra homomorphism $k\left[\left(\mathbb{A}^{n^{2}}\right)^{r}\right]=k\left[\left\{X_{i j}^{l}\right\}_{l=0}^{r-1} i_{i, j=1}\right] \rightarrow k\left[V_{r}\left(G L_{n}\right)\right]$ which is compatible with gradings provided that we define the grading on the left via the formula $\operatorname{deg}\left(X_{i, j}^{l}\right)=p^{l}$. In any event, the graded algebra on the left is connected and hence $k\left[V_{r}(G)\right]$ is connected as well. The general case now follows immediately, since the surjective homomorphism $k\left[V_{r}\left(G L_{n}\right)\right] \rightarrow k\left[V_{r}(G)\right]$ corresponding to a closed embedding $G \hookrightarrow G L_{n}$ is compatible with gradings.

We shall repeatedly use the structure of the cohomology algebra of $\mathbb{G}_{a}$ and $\mathbb{G}_{a(r)}$ as recalled in the following theorem.

Theorem $1.13([\mathrm{CPSvdK}])$. (1) Assume that $p \neq 2$. Then the cohomology algebra $H^{*}\left(\mathbb{G}_{a}, k\right)$ is a tensor product of a polynomial algebra $k\left[x_{1}, x_{2}, \ldots\right]$ in generators $x_{i}$ of degree 2 and an exterior algebra $\Lambda\left(\lambda_{1}, \lambda_{2}, \ldots\right)$ in generators $\lambda_{i}$ of degree one. If 
$p=2$, then $H^{*}\left(\mathbb{G}_{a}, k\right)=k\left[\lambda_{1}, \lambda_{2}, \ldots\right]$ is a polynomial algebra in generators $\lambda_{i}$ of degree 1 (and in this case we set $x_{i}=\lambda_{i}^{2}$ ).

(2) Let $F: \mathbb{G}_{a} \rightarrow \mathbb{G}_{a}$ denote the Frobenius endomorphism. Then $F^{*}\left(x_{i}\right)=x_{i+1}$, $F^{*}\left(\lambda_{i}\right)=\lambda_{i+1}$.

(3) Let $s$ be an element of $k$ and use the same notation $s$ for the endomorphism (multiplication by s) of $\mathbb{G}_{a}$. Then $s^{*}\left(x_{i}\right)=s^{p^{i}} x_{i}, s^{*}\left(\lambda_{i}\right)=s^{p^{i-1}} \lambda_{i}$.

(4) Restriction of $x_{i}$ and $\lambda_{i}$ to $\mathbb{G}_{a(r)}$ is trivial for $i>r$. Denoting the restrictions of $x_{i}$ and $\lambda_{i}$ (for $\left.i \leq r\right)$ to $\mathbb{G}_{a(r)}$ by the same letter, we have

$$
\begin{array}{llrl}
H^{*}\left(\mathbb{G}_{a(r)}, k\right) & =k\left[x_{1}, \ldots, x_{r}\right] \otimes \Lambda\left(\lambda_{1}, \ldots, \lambda_{r}\right), & & p \neq 2, \\
H^{*}\left(\mathbb{G}_{a(r)}, k\right) & =k\left[\lambda_{1}, \ldots, \lambda_{r}\right], & & p=2 .
\end{array}
$$

We can now introduce the homomorphism $\psi$ which will play a key role in both this paper and $[\mathrm{SFB}]$.

Theorem 1.14. For any affine group scheme $G / k$, there is a natural homomorphism of graded commutative $k$-algebras

$$
\psi: H^{e v}(G, k) \rightarrow k\left[V_{r}(G)\right]
$$

which multiplies degrees by $\frac{p^{r}}{2}$.

Proof. For any affine group scheme $G$ over $k$, the canonical element

$$
1 \in V_{r}(G)\left(k\left[V_{r}(G)\right]\right)=\text { Hom }_{k-a l g}\left(k\left[V_{r}(G)\right], k\left[V_{r}(G)\right]\right)
$$

corresponds to a universal homomorphism of group schemes over $k\left[V_{r}(G)\right]$ :

$$
u: \mathbb{G}_{a(r)} \otimes_{k} k\left[V_{r}(G)\right] \rightarrow G \otimes_{k} k\left[V_{r}(G)\right] .
$$

For any commutative $k$-algebra $A$, the $A$-valued point $\left\langle a \in \mathbb{G}_{a(r)}(A), \phi: \mathbb{G}_{a(r)} \otimes_{k}\right.$ $\left.A \rightarrow G \otimes_{k} A\right\rangle$ is sent via $u$ to $\langle\phi(a) \in G(A), \phi\rangle$. This universal homomorphism $u$ induces

$$
\begin{aligned}
u^{*}: H^{*}(G, k) \rightarrow H^{*}(G, k) \otimes_{k} k\left[V_{r}(G)\right] & =H^{*}\left(G \otimes_{k} k\left[V_{r}(G)\right], k\left[V_{r}(G)\right]\right) \\
\rightarrow H^{*}\left(\mathbb{G}_{a(r)} \otimes_{k} k\left[V_{r}(G)\right], k\left[V_{r}(G)\right]\right) & =H^{*}\left(\mathbb{G}_{a(r)}, k\right) \otimes_{k} k\left[V_{r}(G)\right] .
\end{aligned}
$$

For $z \in H^{2 n}(G, k)$, write

$$
u^{*}(z)=\sum_{j, i} \lambda^{j} x^{i} \otimes f_{j, i}(z) \in H^{2 n}\left(\mathbb{G}_{a(r)}, k\right) \otimes k\left[V_{r}(G)\right]
$$

where $\lambda^{j} x^{i}=\lambda_{1}^{j_{1}} \cdots \lambda_{r}^{j_{r}} x_{1}^{i_{1}} \cdots x_{r}^{i_{r}}$ with $j_{\ell} \in\{0,1\}$. We define $\psi(z)$ to be the coefficient of $x_{r}^{n}$ in this expression. That $\psi$ is a homomorphism of commutative $k$-algebras is obvious from the construction.

To verify that $\psi$ is natural, let $h: G \rightarrow G^{\prime}$ be a homomorphism of affine group schemes over $k$ and let $\eta: V_{r}(G) \rightarrow V_{r}\left(G^{\prime}\right)$ denote the induced map of schemes. Then the above explicit description of the universal homomorphism $u$ on $A$-valued points easily implies the commutativity of the following square:

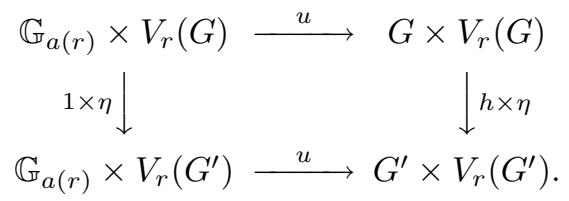

(Namely, $\langle a, \phi\rangle$ maps via $u$ to $\langle\phi(a), \phi\rangle$ which then maps via $h \times \eta$ to $\langle h(\phi(a)), h \circ \phi\rangle$, whereas $\langle a, \phi\rangle$ maps via $1 \times \eta$ to $\langle a, h \circ \phi\rangle$ which then maps via $u$ to $\langle h(\phi(a)), h \circ \phi\rangle$.) 
To verify that $\psi$ multiplies degrees by $\frac{p^{r}}{2}$, it suffices to assume that $k$ is algebraically closed by replacing $k$ by its algebraic closure. The natural monoid action (which we denote by $\cdot$ ) of $\mathbb{A}^{1}$ on $\mathbb{G}_{a(r)}$ is compatible with the action of $\mathbb{A}^{1}$ on $V_{r}(G)$ given above (which we denote by $\mu$ ) in the sense that the following diagram commutes:

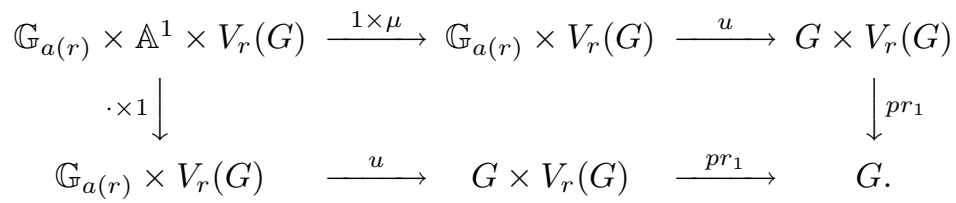

We check this commutativity by considering the associated diagram of $A$-valued points for any commutative $k$-algebra $A$; since $\mu$ is defined in terms of composition of morphisms and was seen above to correspond to the usual multiplication $\cdot$ on $\mathbb{A}^{1}$, this is verified by inspection. Consequently, for any $s \in k$ the following diagram commutes:

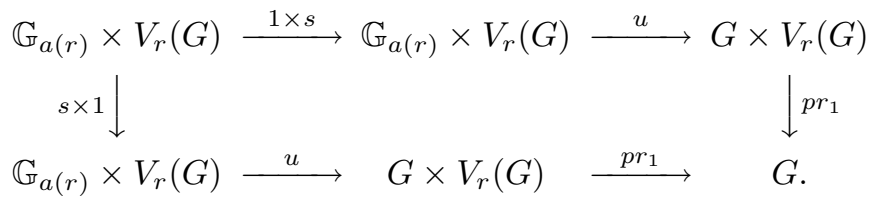

If we pull $z \in H^{2 n}(G, k)$ back via the lower horizontal and left vertical maps, then we obtain

$$
\sum_{j, i} s^{j_{1}+\ldots+p^{r-1} j_{r}+p i_{1}+\ldots+p^{r} i_{r}} \lambda^{j} x^{i} \otimes f_{j, i}
$$

If we pull $z \in H^{2 n}(G, k)$ back via the right vertical and upper horizontal maps, then we obtain

$$
\sum_{j, i} \lambda^{j} x^{i} \otimes s^{*}\left(f_{j, i}\right)
$$

Thus, each $f_{j, i}$ is homogeneous of degree $j_{1}+\ldots+p^{r-1} j_{r}+p i_{1}+\ldots+p^{r} i_{r}$. In particular, $\psi(z)$ has homogeneous degree $p^{r} n$.

In the special case $p=2$, the previous theorem can be refined as follows to include cohomology classes of odd degree.

Theorem 1.14.1. Assume that $p=2$. Then for any affine group scheme $G / k$, there is a natural homomorphism of graded commutative $k$-algebras

$$
\psi: H^{*}(G, k) \rightarrow k\left[V_{r}(G)\right]
$$

which multiplies degrees by $2^{r-1}$.

Proof. This time we define $\psi(z)$ (for $z \in H^{n}(G, k)$ ) to be the coefficient of $\lambda_{r}^{n}$ in $u^{*}(z)$. The properties of this map $\psi$ are verified in exactly the same way as above. 


\section{§2. EXTENSION OF SCALARS IN POLYNOMIAL FUNCTORS}

The theory of strict polynomial functors developed in [F-S] is the basis for the construction of universal extension classes $e_{r}$. Since we need these universal classes in the context of group schemes over quite general commutative rings, we must extend the formulation of strict polynomial functors to this more general situation. We outline below this theory indicating the necessary (minor) changes. We then verify that the universal classes constructed in $[\mathrm{F}-\mathrm{S}]$ (in the context of strict polynomial functors on finite-dimensional vector spaces over a field) determine by base change universal classes in our more general context.

Let $A$ be a commutative ring (always assumed to be unital). Denote by $\mathcal{V}=\mathcal{V}_{A}$ the category of finitely generated projective $A$-modules and $A$-linear maps. For any $V, W \in \mathcal{V}_{A}$ we set $\operatorname{Hom}_{P o l}(V, W)=S^{*}\left(V^{\#}\right) \otimes_{A} W$, where $V^{\#}=\operatorname{Hom}_{A}(V, A)$ is the module dual to $V$ (over $A$ ) and $S^{*}$ stands for the symmetric algebra (over $A$ ). The elements of $\operatorname{Hom}_{P o l}(V, W)$ are called polynomial maps from $V$ to $W$. A polynomial map is said to be homogeneous of degree $d$ if it belongs to $S^{d}\left(V^{\#}\right) \otimes_{A} W$. The set of homogeneous polynomial maps of degree one coincides with $\mathrm{Hom}_{A}(V, W)$. Each bilinear map $U \times V \rightarrow W$ defines a homogeneous polynomial map of degree two.

For any $V \in \mathcal{V}$, we shall use the same notation $V$ for the corresponding vector bundle $S$ pec $S^{*}\left(V^{\#}\right)$ over $S p e c A$. With this notation, we have the following obvious formula:

$$
\operatorname{Hom}_{P o l}(V, W)=H_{S o m} / A(V, W) .
$$

This formula enables us, in particular, to define the composition map

$$
\mathrm{Hom}_{\mathrm{Pol}}(V, W) \times \mathrm{Hom}_{\mathrm{Pol}}(U, V) \rightarrow \mathrm{Hom}_{\mathrm{Pol}}(U, W) .
$$

Each polynomial map $p: V \rightarrow W$ defines a set map from $V$ to $W$ which we usually denote by the same letter $p$. Note also that we can extend scalars for polynomial maps, so that whenever $p: V \rightarrow W$ is a polynomial map (over $A$ ) and $A^{\prime}$ is a commutative $A$-algebra we may consider a polynomial map $p_{A^{\prime}}: V_{A^{\prime}} \rightarrow W_{A^{\prime}}$ over $A^{\prime}$ and hence also the corresponding set map $V_{A^{\prime}} \rightarrow W_{A^{\prime}}$.

Definition 2.1. A strict polynomial functor over a commutative $\operatorname{ring} A, T: \mathcal{V}_{A} \rightarrow$ $\mathcal{V}_{A}$, consists of the data of $T(V) \in \mathcal{V}_{A}$ for each $V \in \mathcal{V}_{A}$ and a polynomial map $T_{V, W}: \operatorname{Hom}_{A}(V, W) \rightarrow \operatorname{Hom}_{A}(T(V), T(W))$ for each pair $V, W \in \mathcal{V}_{A}$, satisfying

(2.1.1) $T_{V, V}\left(1_{V}\right)=1_{T(V)}$.

(2.1.2) For any $U, V, W \in \mathcal{V}_{A}$, the following diagram of polynomial maps commutes:

$$
\begin{array}{rc}
\operatorname{Hom}_{A}(V, W) \times \operatorname{Hom}_{A}(U, V) & \operatorname{Hom}_{A}(U, W) \\
T_{V, W} \times T_{U, V} \downarrow & \downarrow T_{U, W} \\
\operatorname{Hom}_{A}(T(V), T(W)) \times \operatorname{Hom}_{A}(T(U), T(V)) & \longrightarrow \operatorname{Hom}_{A}(T(U), T(W)) .
\end{array}
$$

For any $V \in \mathcal{V}_{A}$, we denote by $G L(V)$ the corresponding affine group scheme over $A$. As a scheme, $G L(V)$ is the principal open subset in the vector bundle $\operatorname{End}_{A}(V)$ given by the invertibility of the determinant $\operatorname{det} \in S^{*}\left(\operatorname{End}_{A}(V)^{\#}\right)$. If $G$ is an affine group scheme of finite type over $A$, then to make $V \in \mathcal{V}_{A}$ into a rational $G$-module (i.e. into a comodule over the coalgebra $A[G]$ ) is equivalent to giving a group scheme homomorphism $G \rightarrow G L(V)$ over $A$. 
Let $T$ be a polynomial functor over $A$. The data of a polynomial functor provides, in particular, a morphism of schemes

$$
T_{V, V}: \operatorname{End}_{A}(V) \rightarrow \operatorname{End}_{A}(T(V)) .
$$

One checks easily that $T_{V, V}$ takes the open subscheme $G L(V) \subset \operatorname{End}_{A}(V)$ to $G L(T(V))$ and the induced morphism of schemes $T_{V, V}: G L(V) \rightarrow G L(T(V))$ is a group scheme homomorphism. Composing this homomorphism with the original homomorphism $G \rightarrow G L(V)$, we see that $T(V)$ has a natural structure of a rational $G$-module.

Now the general theory developed in $[\mathrm{F}-\mathrm{S}], \S 2$, goes through practically without change. We briefly recall the essential definitions and results.

First of all, one can introduce the concept of a homogeneous strict polynomial functor of degree $d$ (resp. of a strict polynomial functor of finite degree) by requiring that all polynomial maps $T_{V, V}: \operatorname{End}_{A}(V) \rightarrow \operatorname{End}_{A}(T(V))$ are homogeneous of degree $d$ (resp. degrees of these maps are bounded above). One checks easily that a strict polynomial functor of finite degree is a direct sum of (finitely many) homogeneous components.

One defines a natural transformation of strict polynomial functors exactly in the same way as was done in $[\mathrm{F}-\mathrm{S}]$ for polynomial functors over a field. We denote by $\mathcal{P}=\mathcal{P}_{A}$ the category of strict polynomial functors over $A$ of finite degree and by $\mathcal{P}_{d}=\mathcal{P}_{d, A}$ its full subcategory consisting of homogeneous strict polynomial functors of degree $d$. As mentioned above, we have a canonical direct sum decomposition $\mathcal{P}_{A}=\bigoplus_{d \geq 0} \mathcal{P}_{d, A}$. In the present situation, the categories $\mathcal{P}_{A}$ and $\mathcal{P}_{d, A}$ are no longer abelian; however, they are exact categories in the sense of Quillen [Q] with admissible short exact sequences $0 \rightarrow T^{\prime} \rightarrow T \rightarrow T^{\prime \prime} \rightarrow 0$ being those for which the sequence $0 \rightarrow T^{\prime}(V) \rightarrow T(V) \rightarrow T^{\prime \prime}(V) \rightarrow 0$ is exact for any $V \in \mathcal{V}_{A}$.

We recall the notation $\Gamma^{i}$ for the $i$-th divided power, the dual (over $A$ ) of $S^{i}$.

Let $T$ be a strict polynomial functor. For any $V, W \in \mathcal{V}_{A}, T$ defines a canonical $A$-linear homomorphism

$$
\theta: \bigoplus_{i \geq 0} \Gamma^{i}\left(H_{o m}(V, W)\right) \otimes_{A} T(V) \rightarrow T(W)
$$

which is nonzero only on finitely many components (only on $\Gamma^{d}\left(H_{o m}(V, W)\right) \otimes_{A}$ $T(V)$ if $T$ is homogeneous of degree $d$ ). A functor $T$ is said to be $n$-generated provided that the corresponding homomorphism

$$
\bigoplus_{i \geq 0} \Gamma^{i}\left(\operatorname{Hom}_{A}\left(A^{n}, W\right)\right) \otimes_{A} T\left(A^{n}\right) \rightarrow T(W)
$$

is surjective for any $W \in \mathcal{V}_{A}$. A functor $T$ is said to be finitely generated if it is $n$-generated for some $n \geq 0$. Arguing essentially in the same way as in [F-S], one can show that $T$ is finitely generated if and only if it is of finite degree. In fact we have the following result.

Proposition 2.2 ([F-S], Proposition 2.9). A strict polynomial functor T is finitely generated if and only if it is of finite degree. More precisely, if $T$ is $n$-generated, then deg $(T)$ coincides with the degree of the polynomial map $T_{A^{n}, A^{n}}: \operatorname{End}_{A}\left(A^{n}\right) \rightarrow$ $\operatorname{End}_{A}\left(T\left(A^{n}\right)\right)$; conversely if $T$ has degree $d$, then it is d-generated.

The functor $\Gamma^{d, n} \in \mathcal{P}_{d}$ introduced in [F-S] (see also the proof of Proposition 3.6) is given by the formula $\Gamma^{d, n}(V)=\Gamma^{d}\left(\operatorname{Hom}_{A}\left(A^{n}, V\right)\right)$. This functor is relatively projective as one sees from the following result. 
Theorem 2.3 ([F-S], Theorem 2.10). For any homogeneous strict polynomial functor of degree $d$ and any $n \geq 0$,

$$
\operatorname{Hom}_{\mathcal{P}_{d}}\left(\Gamma^{d, n}, T\right)=T\left(A^{n}\right) .
$$

Thus, $\Gamma^{d, n}$ is a relatively projective object of $\mathcal{P}_{d}$. Moreover, for any homogeneous strict polynomial functor $T$ of degree $d$, the associated natural transformation

$$
\Theta: \Gamma^{d, n} \otimes_{A} T\left(A^{n}\right) \rightarrow T
$$

is an admissible epimorphism if and only if $T$ is n-generated.

Theorem 2.3 and Proposition 2.2 show that each $T \in \mathcal{P}_{d}$ has an admissible projective resolution. Thus we may develop the usual relative homological algebra in the category $\mathcal{P}_{d}$. In particular, for any $T, T^{\prime} \in \mathcal{P}_{d}$ we may consider the corresponding Ext-groups $\operatorname{Ext} t_{\mathcal{P}}^{*}\left(T, T^{\prime}\right)$.

Denote by $S(n, d)=S(n, d, A)$ the Schur algebra $\Gamma^{d}\left(M_{n}(A)\right)$. For any $T \in \mathcal{P}_{d}$ and any $n \geq 0$, the canonical homomorphism

$$
\theta: \Gamma^{d}\left(\operatorname{End}_{A}\left(A^{n}\right)\right) \otimes_{A} T\left(A^{n}\right) \rightarrow T\left(A^{n}\right)
$$

makes $T\left(A^{n}\right)$ into a finitely generated left $S(n, d, A)$-module, which is projective as an $A$-module. Denote by $\operatorname{Mod}\{S(n, d, A)\}$ the exact category of all such modules. Associating to $T \in \mathcal{P}_{d}$ the $S(n, d, A)$-module $T\left(A^{n}\right)$, we get an exact functor

$$
\Psi: \mathcal{P}_{d} \rightarrow \operatorname{Mod}\{S(n, d, A)\}
$$

Theorem 2.4 ([F-S], Theorem 3.2). Assume that $n \geq d$. Then

$$
\Psi: \mathcal{P}_{d, A} \rightarrow \operatorname{Mod}\{S(n, d, A)\}
$$

is an equivalence of categories with quasi-inverse defined by sending $M \in$ $\operatorname{Mod}\{S(n, d, A)\}$ to $\Phi(M) \equiv \Gamma^{d, n} \otimes_{S(n, d, A)} M$.

The proof is identical with that given in [F-S], the only additional problem being the verification that if $M \in \operatorname{Mod}\{S(n, d, A)\}$ and $V \in \mathcal{V}_{A}$, then $\Gamma^{d, n}(V) \otimes_{S(n, d, A)} M$ is a projective $A$-module. To check this, it suffices to consider $V=A^{m}$. In this case, one can use the fact that $\Gamma^{d, n}\left(A^{m}\right)=\Gamma^{d}\left(\operatorname{Hom}_{A}\left(A^{n}, A^{m}\right)\right)$ is a projective right $S(n, d, A)$-module.

Corollary 2.4.1. Let $f: T \rightarrow T^{\prime}$ be a homomorphism of homogeneous strict polynomial functors of degree $d$. The following conditions are equivalent:

(a) $f$ is an isomorphism.

(b) $f_{A^{n}}: T\left(A^{n}\right) \rightarrow T^{\prime}\left(A^{n}\right)$ is an isomorphism for all $n$.

(c) $f_{A^{n}}: T\left(A^{n}\right) \rightarrow T^{\prime}\left(A^{n}\right)$ is an isomorphism for some $n \geq d$.

We now investigate the behavior of Ext-groups under base change. Let $A^{\prime}$ be a commutative $A$-algebra and let $T \in \mathcal{P}_{A}, T^{\prime} \in \mathcal{P}_{A^{\prime}}$ be a pair of strict polynomial functors. We define a homomorphism $f: T \rightarrow T^{\prime}$ to be a collection of $A^{\prime}$-linear maps

$$
f_{V}: T(V) \otimes_{A} A^{\prime} \rightarrow T^{\prime}\left(V \otimes_{A} A^{\prime}\right)
$$


given for all $V \in \mathcal{V}_{A}$ and satisfying the following property: for any $V, W \in \mathcal{V}_{A}$, the following diagram of polynomial maps commutes:

$$
\begin{array}{ccc}
\operatorname{Hom}_{A^{\prime}}\left(V_{A^{\prime}}, W_{A^{\prime}}\right)=\left(\operatorname{Hom}_{A}(V, W)\right)_{A^{\prime}} \stackrel{\left(T_{V, W}\right)_{A^{\prime}}}{\longrightarrow} \operatorname{Hom}_{A^{\prime}}\left(T(V)_{A^{\prime}}, T(W)_{A^{\prime}}\right) \\
T_{V_{A^{\prime}}, W_{A^{\prime}} \downarrow} \downarrow & f_{W^{\circ}} \\
\operatorname{Hom}_{A^{\prime}}\left(T^{\prime}\left(V_{A^{\prime}}\right), T^{\prime}\left(W_{A^{\prime}}\right)\right) & \stackrel{\circ f_{V}}{\longrightarrow} \operatorname{Hom}_{A^{\prime}}\left(T(V)_{A^{\prime}}, T^{\prime}\left(W_{A^{\prime}}\right)\right) .
\end{array}
$$

We have used here and below the notation $V_{A^{\prime}}$ to denote $V \otimes_{A} A^{\prime}$ for any $V \in \mathcal{V}_{A}$.

Let $V^{\prime} \in \mathcal{V}_{A^{\prime}}$ and consider the category $\mathcal{V}_{A}\left(V^{\prime}\right)$, whose objects are pairs $(V, \phi)$, where $V \in \mathcal{V}_{A}$ and $\phi: V \otimes_{A} A^{\prime} \rightarrow V^{\prime}$ is an $A^{\prime}$-linear map, and morphisms from $(V, \phi)$ to $\left(V_{1}, \phi_{1}\right)$ are $A^{\prime}$-linear maps $\psi: V \otimes_{A} A^{\prime} \rightarrow V_{1} \otimes_{A} A^{\prime}$ such that $\phi_{1} \psi=\phi$. Let $T \in \mathcal{P}_{A}$ be a strict polynomial functor. To each $(V, \phi) \in \mathcal{V}_{A}\left(V^{\prime}\right)$ we associate the $A^{\prime}$-module $T(V)_{A^{\prime}}=T(V) \otimes_{A} A^{\prime}$, and to each $\psi \in \operatorname{Hom}\left((V, \phi),\left(V_{1}, \phi_{1}\right)\right)$ we associate the $A^{\prime}$-linear map

$$
\left(T_{V, V_{1}}\right)_{A^{\prime}}(\psi): T(V)_{A^{\prime}} \rightarrow T\left(V_{1}\right)_{A^{\prime}} .
$$

In this way, we get a functor from the category $\mathcal{V}_{A}\left(V^{\prime}\right)$ to the category of $A^{\prime}$ modules. We define the $A^{\prime}$-module $T_{A^{\prime}}\left(V^{\prime}\right)$ via the formula

$$
T_{A^{\prime}}\left(V^{\prime}\right)=\underset{\mathcal{V}_{A}\left(V^{\prime}\right)}{\lim } T(V)_{A^{\prime}}
$$

Observe that

$$
T_{A^{\prime}}\left(V_{A^{\prime}}\right)=T(V)_{A^{\prime}}, \quad V \in \mathcal{V}_{A},
$$

since $\left(V, 1_{V_{A^{\prime}}}\right)$ is the final object of the category $\mathcal{V}_{A}\left(V_{A^{\prime}}\right)$.

Proposition 2.5. Let $T \in \mathcal{P}_{A}$ and let $A^{\prime}$ be a commutative A-algebra. Then the functor $T_{A^{\prime}}$ sending $V^{\prime} \in \mathcal{V}_{A^{\prime}}$ to $T_{A^{\prime}}\left(V^{\prime}\right)=\underset{\mathcal{V}_{A}\left(V^{\prime}\right)}{\lim } T(V)_{A^{\prime}}$ is a strict polynomial functor on $\mathcal{V}_{A^{\prime}}$. Moreover, for any $T^{\prime} \in \mathcal{P}_{A^{\prime}}$,

$$
\operatorname{Hom}_{\mathcal{P}_{A}}\left(T, T^{\prime}\right)=H_{o m} \bar{P}_{A^{\prime}}\left(T_{A^{\prime}}, T^{\prime}\right) .
$$

Proof. It's clear that the so-defined $T_{A^{\prime}}$ is at least a functor in the usual sense from $\mathcal{V}_{A^{\prime}}$ to the category of $A^{\prime}$-modules. Furthermore, for any $V^{\prime} \in \mathcal{V}_{A^{\prime}}$ we can find an embedding $i: V^{\prime} \hookrightarrow A^{\prime n}$ and a projection $p: A^{\prime n} \rightarrow V^{\prime}$ for which $p \circ i=1_{V^{\prime}}$. This implies that $T_{A^{\prime}}(p) \circ T_{A^{\prime}}(i)=1_{T_{A^{\prime}}\left(V^{\prime}\right)}$ and hence $T_{A^{\prime}}\left(V^{\prime}\right)$ is a direct summand in $T_{A^{\prime}}\left(A^{\prime n}\right)=T\left(A^{n}\right)_{A^{\prime}}$ and in particular $T_{A^{\prime}}\left(V^{\prime}\right) \in \mathcal{V}_{A^{\prime}}$.

To define polynomial maps

$$
\left(T_{A^{\prime}}\right)_{V^{\prime}, W^{\prime}}: \operatorname{Hom}_{A^{\prime}}\left(V^{\prime}, W^{\prime}\right) \rightarrow \operatorname{Hom}_{A^{\prime}}\left(T_{A^{\prime}}\left(V^{\prime}\right), T_{A^{\prime}}\left(W^{\prime}\right)\right)
$$

we proceed as follows. Choose embeddings $i_{V^{\prime}}: V^{\prime} \hookrightarrow A^{\prime n}, i_{W^{\prime}}: W^{\prime} \hookrightarrow A^{\prime m}$ and projections $p_{V^{\prime}}: A^{\prime n} \rightarrow V^{\prime}, p_{W^{\prime}}: A^{\prime m} \rightarrow W^{\prime}$ such that $p_{V^{\prime}} i_{V^{\prime}}=1_{V^{\prime}}, p_{W^{\prime}} i_{W^{\prime}}=$ $1_{W^{\prime}}$ and define $\left(T_{A^{\prime}}\right)_{V^{\prime}, W^{\prime}}$ as a composition

$$
\begin{gathered}
\operatorname{Hom}_{A^{\prime}}\left(V^{\prime}, W^{\prime}\right) \rightarrow \operatorname{Hom}_{A^{\prime}}\left(A^{\prime n}, A^{\prime m}\right) \stackrel{\left(T_{\left.A^{n}, A^{m}\right)_{A^{\prime}}}\right.}{\longrightarrow} \operatorname{Hom}_{A^{\prime}}\left(T\left(A^{n}\right)_{A^{\prime}}, T\left(A^{m}\right)_{A^{\prime}}\right) \\
=\operatorname{Hom}_{A^{\prime}}\left(T_{A^{\prime}}\left(A^{\prime \prime}\right), T_{A^{\prime}}\left(A^{\prime m}\right)\right) \rightarrow \operatorname{Hom}_{A^{\prime}}\left(T_{A^{\prime}}\left(V^{\prime}\right), T_{A^{\prime}}\left(W^{\prime}\right)\right) .
\end{gathered}
$$

A routine verification shows that the resulting polynomial map is well defined and that $T_{A^{\prime}}$ is a strict polynomial functor over $A^{\prime}$ having the required universal mapping property. 
Proposition 2.6. The extension of scalars functor $\otimes_{A} A^{\prime}: \mathcal{P}_{A} \rightarrow \mathcal{P}_{A^{\prime}}$ sending $T$ to $T_{A^{\prime}}$ is exact and preserves (relative) projectives. Furthermore,

$$
\operatorname{Hom}_{\mathcal{P}_{A^{\prime}}}\left(S_{A^{\prime}}, T_{A^{\prime}}\right)=\operatorname{Hom}_{\mathcal{P}_{A}}(S, T) \otimes_{A} A^{\prime}, \quad S, T \in \mathcal{P}_{A} .
$$

Proof. Let $0 \rightarrow T^{1} \rightarrow T \rightarrow T^{2} \rightarrow 0$ be an exact sequence in $\mathcal{P}_{A}$. Let further $V^{\prime}$ be an object of $\mathcal{V}_{A^{\prime}}$. If $V^{\prime}$ is of the form $V^{\prime}=V_{A^{\prime}}$, then (2.5.0) implies that the sequence

$$
0 \rightarrow T_{A^{\prime}}^{1}\left(V^{\prime}\right) \rightarrow T_{A^{\prime}}\left(V^{\prime}\right) \rightarrow T_{A^{\prime}}^{2}\left(V^{\prime}\right) \rightarrow 0
$$

is isomorphic to

$$
0 \rightarrow T^{1}(V) \otimes_{A} A^{\prime} \rightarrow T(V) \otimes_{A} A^{\prime} \rightarrow T^{2}(V) \otimes_{A} A^{\prime} \rightarrow 0
$$

and hence is exact. In the general case we may choose a split embedding of $V^{\prime}$ into $A^{\prime n}$. Then the sequence (2.6.1) is isomorphic to a direct summand in an exact sequence corresponding to $A^{\prime n}$ and hence is again exact.

To show that $\otimes_{A} A^{\prime}$ preserves projectives it suffices to establish the formula $\left(\Gamma_{A}^{d, n}\right) \otimes_{A} A^{\prime}=\Gamma_{A^{\prime}}^{d, n}$, which is done by direct inspection.

In proving the last statement, we may clearly assume that $S$ and $T$ are homogeneous of degree $d$. If $S=\Gamma^{d, n}$, then our statement follows from Theorem 2.3 and (2.5.0). For a general $S$, we may consider a resolution of the form

$$
\left(\Gamma^{d, d}\right)^{m} \rightarrow\left(\Gamma^{d, d}\right)^{n} \rightarrow S \rightarrow 0
$$

and use the right exactness of the functor Hom.

Proposition 2.6 has the following formal consequence.

Corollary 2.7. For any $S, T \in \mathcal{P}_{A}$, we have a canonical homomorphism

$$
\operatorname{Ext}_{\mathcal{P}_{A}}^{*}(S, T) \otimes_{A} A^{\prime} \rightarrow \operatorname{Ext}_{\mathcal{P}_{A^{\prime}}}^{*}\left(S_{A^{\prime}}, T_{A^{\prime}}\right)
$$

which is an isomorphism in case $A^{\prime}$ is flat over $A$.

We shall be particularly interested in the base change of the functors $I^{(r)}$ whose Ext-algebra was determined in [F-S]. For any commutative $\mathbb{F}_{p}$-algebra $A$, let $f$ : $A \rightarrow A$ denote the Frobenius ( $p$-th power) map sending $a \in A$ to $a^{p} \in A$. For any $V \in \mathcal{V}_{A}$ and any $r \geq 0$, set $V^{(r)}=V \otimes_{f^{r}} A$. The functor $I^{(r)}=I_{A}^{(r)}: V \mapsto V^{(r)}$ is an additive functor from the category $\mathcal{V}_{A}$ to itself satisfying the following properties:

$$
\left(V \otimes_{A} W\right)^{(r)}=V^{(r)} \otimes_{A} W^{(r)} ; \quad\left(V^{\#}\right)^{(r)}=\left(V^{(r)}\right)^{\#} \quad \text { for any } V, W \in \mathcal{V}_{A} .
$$

We'll be using the notation $v^{(r)}$ for the element $v \otimes_{f^{r}} 1 \in V^{(r)}$. It's clear that if $V$ is free with basis $v_{1}, \ldots, v_{n}$, then $V^{(r)}$ is also free with basis $v_{1}^{(r)}, \ldots, v_{n}^{(r)}$. To make $I^{(r)}$ into a polynomial functor, we note that for any $V \in \mathcal{V}_{A}$ we have a canonical $A$ linear homomorphism $V^{(r)} \rightarrow S^{p^{r}}(V): v^{(r)} \mapsto v^{p^{r}}$. Applying this to $V^{\#}$ we get a canonical polynomial map $V \rightarrow V^{(r)}$ of degree $p^{r}$ (the corresponding set map takes $v$ to $\left.v^{(r)}\right)$. In particular, this gives us for any $V, W \in \mathcal{V}_{A}$ a canonical polynomial map

$$
\operatorname{Hom}_{A}(V, W) \rightarrow \operatorname{Hom}_{A}(V, W)^{(r)}=\operatorname{Hom}_{A}\left(V^{(r)}, W^{(r)}\right) .
$$

A straightforward verification shows that with these maps $I^{(r)}$ becomes a homogeneous polynomial functor of degree $p^{r}$ over $A$. We sum up the results of the previous discussion in the following proposition. 
Proposition 2.8. Let $A$ be a commutative $\mathbb{F}_{p}$-algebra. Then $I_{A}^{(r)}$ is a homogeneous polynomial functor of degree $p^{r}$. Moreover $I_{A}^{(r)}$ is additive and satisfies the following properties:

$$
\left(V \otimes_{A} W\right)^{(r)}=V^{(r)} \otimes_{A} W^{(r)} ; \quad\left(V^{\#}\right)^{(r)}=\left(V^{(r)}\right)^{\#} \quad \text { for any } V, W \in \mathcal{V}_{A} .
$$

Finally for any $A$ as above we have a canonical isomorphism

$$
I_{A}^{(r)}=I_{\mathbb{F}_{p}}^{(r)} \otimes_{\mathbb{F}_{p}} A .
$$

Proof. Everything but the last statement has been established above. To prove the last statement we first mention that for any $V_{0} \in \mathcal{V}_{\mathbb{F}_{p}}$ we have a canonical isomorphism

$$
V_{0}^{(r)} \otimes_{\mathbb{F}_{p}} A \stackrel{\sim}{\longrightarrow}\left(V_{0} \otimes_{\mathbb{F}_{p}} A\right)^{(r)} .
$$

One checks easily that these isomorphisms define a homomorphism $I_{\mathbb{F}_{p}}^{(r)} \rightarrow I_{A}^{(r)}$. The universal mapping property of Proposition 2.5 gives us further a canonical functor homomorphism $I_{\mathbb{F}_{p}}^{(r)} \otimes_{\mathbb{F}_{p}} A \rightarrow I_{A}^{(r)}$. The formula (2.5.0) shows that the corresponding $A$-linear map

$$
\left(I_{\mathbb{F}_{p}}^{(r)} \otimes_{\mathbb{F}_{p}} A\right)(V) \rightarrow I_{A}^{(r)}(V)
$$

is an isomorphism for modules of the form $V=V_{0} \otimes_{\mathbb{F}_{p}} A \quad\left(V_{0} \in \mathcal{V}_{\mathbb{F}_{p}}\right)$, i.e. for free $A$-modules. To complete the proof, it suffices now to use Corollary 2.4.1.

\section{§3. Characteristic Classes and the Hopf algebra $E_{r}$}

In $[\mathrm{F}-\mathrm{S}]$, the determination of the (Yoneda) Ext-algebra $E_{r}=\operatorname{Ext}_{\mathcal{P}_{\mathbb{F}_{p}}}^{*}\left(I^{(r)}, I^{(r)}\right)$ played an important role. In this section, we view elements of this algebra as universal characteristic classes for rational representations. We also introduce a natural coproduct on $E_{r}$ which provides $E_{r}$ with the structure of a commutative, cocommuative Hopf algebra (whose explicit form will be determined in the next section).

The following computation of $E_{r}$ was achieved in [F-S].

Theorem 3.1 ([F-S], Theorem 4.10). Each $E_{r}$ is a finite-dimensional, commutative $\mathbb{F}_{p}$-algebra concentrated in even degrees. Frobenius twist determines an injective map $E_{r-1} \rightarrow E_{r}$ for each $r>0$. Moreover, $E_{0}=\mathbb{F}_{p}$ (in degree 0 ) and $E_{r}$ is obtained from $E_{r-1}$ by adjoining a new generator

$$
e_{r} \in \operatorname{Ext}_{\mathcal{P}_{\mathbb{F}_{p}}}^{2 p^{r-1}}\left(I^{(r)}, I^{(r)}\right)
$$

subject to the only relation $e_{r}^{p}=0$. In particular, the dimension of $E_{r}$ in degree $n$ is 1 if $n=2 j, 0 \leq j<p^{r}$, and is 0 otherwise. If $j=j_{0}+j_{1} p+\ldots+j_{r-1} p^{r-1}$ with each $j_{i}$ satisfying $0 \leq j_{i}<p$, then we define

$$
e_{r}(j)=\frac{\left(e_{1}^{(r-1)}\right)^{j_{0}}\left(e_{2}^{(r-2)}\right)^{j_{1}} \ldots e_{r}^{j_{r-1}}}{\left(j_{0} !\right)\left(j_{1} !\right) \cdots\left(j_{r-1} !\right)} \in \operatorname{Ext}_{\mathcal{P}_{\mathbb{F}_{p}}}^{2 j}\left(I^{(r)}, I^{(r)}\right) .
$$

(Thus, $e_{r}=e_{r}\left(p^{r-1}\right)$.)

In the following proposition, we introduce characteristic classes for rational $G$ modules obtained from the universal classes $e_{r}$ of Theorem 3.1. 
Proposition 3.2. Let $G$ be an affine group scheme over a commutative $\mathbb{F}_{p}$-algebra $A$ and let $V \in \mathcal{V}_{A}$ be a rational $G$-module with structure provided by $\rho: G \rightarrow G L(V)$. For each $r>0$, this data determines the characteristic class

$$
e_{r}(G, V) \in \operatorname{Ext}_{G}^{2 p^{r-1}}\left(V^{(r)}, V^{(r)}\right)
$$

(and characteristic classes $e_{r}(j)(G, V) \in \operatorname{Ext}_{G}^{2 j}\left(V^{(r)}, V^{(r)}\right)$ for $\left.j<p^{r}\right)$.

Moreover,

(a) If $\phi: V \rightarrow W$ is a homomorphism of rational $G$-modules, then

$$
\phi^{(r)} \cdot e_{r}(G, V)=e_{r}(G, W) \cdot \phi^{(r)} \in \operatorname{Ext}_{G}^{2 p^{r-1}}\left(V^{(r)}, W^{(r)}\right) .
$$

(b) $e_{r}\left(G, V_{1} \oplus V_{2}\right)=e_{r}\left(G, V_{1}\right) \oplus e_{r}\left(G, V_{2}\right)$.

(c) If $A^{\prime}$ is a commutative A-algebra, then $e_{r}\left(G_{A^{\prime}}, V_{A^{\prime}}\right)=e_{r}(G, V)_{A^{\prime}}$.

Proof. Sending $F \in \mathcal{P}_{\mathbb{F}_{p}}$ to $F_{A}(V) \in$ (rational $G L(V)$-modules) is an exact functor and thereby determines

$$
\begin{aligned}
\operatorname{Ext}_{\mathcal{P}_{\mathbb{F}_{p}}}^{*}\left(F, F^{\prime}\right) & \stackrel{\otimes_{\mathbb{F}_{p}} A}{\longrightarrow} \operatorname{Ext}_{\mathcal{P}_{A}}^{*}\left(F_{A}, F_{A}^{\prime}\right) \rightarrow \operatorname{Ext}_{G L(V)}^{*}\left(F_{A}(V), F_{A}^{\prime}(V)\right) \\
& \rightarrow \operatorname{Ext}_{G}^{*}\left(F_{A}(V), F_{A}^{\prime}(V)\right) .
\end{aligned}
$$

We define $e_{r}(G, V)$ to be the image of $e_{r}$ under this map (with $F=F^{\prime}=I^{(r)}$ ). Let

$$
0 \rightarrow I^{(r)} \rightarrow T_{1} \rightarrow \cdots \rightarrow T_{2 p^{r-1}} \rightarrow I^{(r)} \rightarrow 0
$$

be an exact sequence in $\mathcal{P}_{\mathbb{F}_{p}}$ representing $e_{r}$. Then (a) and (b) are consequences of the following commutative diagrams of rational $G$-modules:

$$
\begin{aligned}
& 0 \rightarrow V^{(r)} \longrightarrow\left(T_{1}\right)_{A}(V) \longrightarrow \cdots \rightarrow\left(T_{2 p^{r-1}}\right)_{A}(V) \longrightarrow V^{(r)} \rightarrow 0 \\
& \phi^{(r)} \downarrow \quad\left(T_{1}\right)_{A}(\phi) \downarrow \quad\left(T_{\left.2 p^{r-1}\right)_{A}(\phi) \downarrow} \downarrow \downarrow^{(r)}\right. \\
& 0 \rightarrow W^{(r)} \longrightarrow\left(T_{1}\right)_{A}(W) \longrightarrow \cdots \rightarrow\left(T_{2 p^{r-1}}\right)_{A}(W) \longrightarrow W^{(r)} \rightarrow 0 \\
& 0 \rightarrow V_{1}^{(r)} \oplus V_{2}^{(r)} \longrightarrow\left(T_{1}\right)_{A}\left(V_{1}\right) \oplus\left(T_{1}\right)_{A}\left(V_{2}\right) \longrightarrow \cdots \rightarrow V_{1}^{(r)} \oplus V_{2}^{(r)} \rightarrow 0 \\
& \left(i_{1}^{(r)}, i_{2}^{(r)}\right) \downarrow \cong \quad\left(\left(T_{1}\right)_{A}\left(i_{1}\right),\left(T_{1}\right)_{A}\left(i_{2}\right)\right) \downarrow \quad\left(i_{1}^{(r)}, i_{2}^{(r)}\right) \downarrow \cong \\
& 0 \rightarrow\left(V_{1} \oplus V_{2}\right)^{(r)} \longrightarrow\left(T_{1}\right)_{A}\left(V_{1} \oplus V_{2}\right) \quad \longrightarrow \cdots \rightarrow\left(V_{1} \oplus V_{2}\right)^{(r)} \rightarrow 0 \text {. }
\end{aligned}
$$

The last statement follows immediately from definitions and formula (2.5.0).

Remark 3.3. Let $G / k$ be an infinitesimal group scheme of height $\leq r$. Let further $A$ be a commutative $k$-algebra and $V \in \mathcal{V}_{A}$ be a rational $G_{A}$-module. In this case the action of $G_{A}$ on $V^{(r)}$ is trivial and hence

$$
\begin{aligned}
\operatorname{Ext}_{G_{A}}^{*}\left(V^{(r)}, V^{(r)}\right) & =\operatorname{End}_{A}\left(V^{(r)}\right) \otimes_{A} H^{*}\left(G_{A}, A\right) \\
& =\operatorname{End}_{A}(V)^{(r)} \otimes_{A} H^{*}\left(G_{A}, A\right)=\operatorname{End}_{A}(V)^{(r)} \otimes_{k} H^{*}(G, k) .
\end{aligned}
$$

Assume further that the module $V$ is free and moreover we have a fixed basis of this module, so that $V$ may be identified with $A^{n}$ and $\operatorname{End}_{A}(V)$ may be identified with the matrix algebra $M_{n}(A)$. The $A$-algebra $M_{n}(A)^{(r)}$ may be further identified with $M_{n}(A)$, this identification takes $\alpha^{(r)}\left(\alpha \in M_{n}(A)\right)$ to a matrix (which we also 
denote $\alpha^{(r)}$ ) whose entries are obtained from those of $\alpha$ by raising them to the power $p^{r}$. In the above situation, we get an isomorphism

$$
\operatorname{Ext}_{G_{A}}^{*}\left(V^{(r)}, V^{(r)}\right)=M_{n}(A) \otimes_{k} H^{*}(G, k)=M_{n}\left(A \otimes_{k} H^{*}(G, k)\right) .
$$

Moreover this isomorphism transforms the Yoneda pairing in $\operatorname{Ext}_{G_{A}}^{*}\left(V^{(r)}, V^{(r)}\right)$ to the usual matrix multiplication in $M_{n}\left(A \otimes_{k} H^{*}(G, k)\right)$.

The isomorphism above enables us to interpret the characteristic classes $e_{r}(j)\left(G_{A}, V\right)$ as matrices with coefficients in the commutative ring $A \otimes_{k} H^{e v}(G, k)=$ $H^{e v}\left(G_{A}, A\right)$. We shall consistently use this point of view in the next section.

Convention 3.4. We adopt the following convention to fix the generators $e_{r}$ (uniquely defined up to scalar multiple). This convention, possibly differing from that of [F-S], is chosen to insure the validity of Proposition 4.1.

Recall (cf. $[\mathrm{F}-\mathrm{P}]$ ) the May spectral sequence

$$
E_{2}^{2 s, t}=H^{t}\left(g l_{n}, k\right) \otimes S^{s}\left(g l_{n}^{(1) \#}\right) \Rightarrow H^{2 s+t}\left(G L_{n(1)}, k\right),
$$

where $H^{*}\left(g l_{n}, k\right)$ denotes Lie algebra cohomology of $g l_{n}=\operatorname{Lie}\left(G L_{n}\right)$. The composition of the natural map $g l_{n}^{(r) \#} \rightarrow S^{p^{r-1}}\left(g l_{n}^{(1) \#}\right)$ and the edge homomorphism of this spectral sequence determines a class in

$$
\operatorname{Hom}\left(g l_{n}^{(r) \#}, H^{2 p^{r-1}}\left(G L_{n(1)}, k\right)\right) \simeq H^{2 p^{r-1}}\left(G L_{n(1)}, g l_{n}^{(r)}\right)
$$

We define $e_{r}$ (i.e., specify the scalar multiple) by requiring that $e_{r}\left(G L_{n(1)}, k^{n}\right)$ equal the preceding class. To make this definition work we have to check that (with a definition of $e_{r}$ used in [F-S]) the class $e_{r}\left(G L_{n(1)}, k^{n}\right) \in H^{2 p^{r-1}}\left(G L_{n(1)}, g l_{n}^{(r)}\right)$ differs from the preceding class by a nonzero constant which is independent of $n$ and $k$. This follows directly from properties of $e_{r}$ given in [F-S], 1.7, 6.1, the first being equality up to nonzero scalar mulitple and the second being compatibility with respect to change of $n$.

The following definition is a natural extension of the definition of a strict polynomial functor. For simplicity, we work over a field $k$, although the definition can be immediately generalized to the case of an arbitrary commutative base ring. For the remainder of this section, we set $\mathcal{P}=\mathcal{P}_{k}$.

Definition 3.5. Let $k$ be a field and $\mathcal{V}=\mathcal{V}_{k}$. A strict polynomial bifunctor

$$
T: \mathcal{V} \times \mathcal{V} \rightarrow \mathcal{V}
$$

consists of the data of $T(V, W) \in \mathcal{V}$ for each pair $(V, W) \in \mathcal{V}^{2}$ and a polynomial map

$$
T_{V_{1}, W_{1} ; V_{2}, W 2}: \operatorname{Hom}_{k}\left(V_{1}, V_{2}\right) \times \operatorname{Hom}_{k}\left(W_{1}, W_{2}\right) \rightarrow \operatorname{Hom}_{k}\left(T\left(V_{1}, W_{1}\right), T\left(V_{2}, W_{2}\right)\right)
$$

for all $\left(V_{1}, W_{1} ; V_{2}, W_{2}\right) \in \mathcal{V}^{4}$ satisfying

(3.5.1) $T_{V, W ; V, W}\left(1_{V}, 1_{W}\right)=1_{T(V, W)}$. 
(3.5.2) For any $\left(V_{1}, W_{1} ; V_{2}, W_{2} ; V_{3}, W_{3}\right) \in \mathcal{V}^{6}$, the following diagram of polynomial maps commutes:

$$
\begin{aligned}
& {\left[V_{2}, V_{3}\right] \times\left[W_{2}, W_{3}\right] \times\left[V_{1}, V_{2}\right] \times\left[W_{1}, W_{2}\right] \quad \longrightarrow \quad\left[V_{1}, V_{3}\right] \times\left[W_{1}, W_{3}\right]} \\
& T_{V_{2}, W_{2} ; V_{3}, W_{3} \times T_{V_{1}, W_{1} ; V_{2}, W_{2}} \downarrow} \downarrow T_{V_{1}, W_{1} ; V_{3}, W_{3}} \\
& {\left[T\left(V_{2}, W_{2}\right), T\left(V_{3}, W_{3}\right)\right] \times\left[T\left(V_{1}, W_{1}\right), T\left(V_{2}, W_{2}\right)\right] \longrightarrow\left[T\left(V_{1}, W_{1}\right), T\left(V_{3}, W_{3}\right)\right]}
\end{aligned}
$$

where we have used $[-,-]$ to denote $\operatorname{Hom}_{k}(-,-)$.

Such a strict polynomial bifunctor $T$ is said to be homogeneous of bidegree $\left(d, d^{\prime}\right)$ provided that $T_{V, W ; V^{\prime}, W^{\prime}}$ is homogeneous of bidegree $\left(d, d^{\prime}\right)$ for all $\left(V, W ; V^{\prime}, W^{\prime}\right) \in$ $\mathcal{V}^{4}$.

We denote by $\mathcal{P}(2)$ the abelian category whose objects are strict polynomial bifunctors of finite degree and whose maps are the evident analogues of natural transformations. Then $\mathcal{P}(2)$ is a direct sum of abelian categories $\mathcal{P}(2)_{d, d^{\prime}}$ of bifunctors of bidegree $\left(d, d^{\prime}\right)$ each with enough projectives and injectives. (Indeed, projective generators in $\mathcal{P}(2)_{d, d^{\prime}}$ are described in the proof of Proposition 3.6 below.)

We shall consider the following constructions of bifunctors from functors:

$$
\begin{gathered}
\odot: \mathcal{P} \times \mathcal{P} \rightarrow \mathcal{P}(2),(F \odot G)(V, W)=F(V) \otimes G(W), \\
\Delta: \mathcal{P} \rightarrow \mathcal{P}(2), \Delta F(V, W)=F(V \otimes W) .
\end{gathered}
$$

Clearly, $\odot$ is biexact and $\Delta$ is exact, so that these functors induce natural maps

$$
\begin{gathered}
\odot^{*}: \operatorname{Ext}_{\mathcal{P}}^{s}(F, G) \otimes \operatorname{Ext}_{\mathcal{P}}^{t}\left(F^{\prime}, G^{\prime}\right) \rightarrow \operatorname{Ext}_{\mathcal{P}(2)}^{s+t}\left(F \odot F^{\prime}, G \odot G^{\prime}\right), \\
\Delta^{*}: \operatorname{Ext}_{\mathcal{P}}^{*}(F, G) \rightarrow \operatorname{Ext}_{\mathcal{P}(2)}^{*}(\Delta(F), \Delta(G)) .
\end{gathered}
$$

Proposition 3.6. For any $F, G \in \mathcal{P}_{d}, F^{\prime}, G^{\prime} \in \mathcal{P}_{d^{\prime}}$,

$$
\odot^{*}: \operatorname{Ext}_{\mathcal{P}}^{*}(F, G) \otimes \operatorname{Ext}_{\mathcal{P}}^{*}\left(F^{\prime}, G^{\prime}\right) \rightarrow \operatorname{Ext}_{\mathcal{P}(2)}^{*}\left(F \odot F^{\prime}, G \odot G^{\prime}\right)
$$

is an isomorphism of graded vector spaces.

Proof. We recall the strict polynomial functor $\Gamma^{d, n} \in \mathcal{P}_{d}$ defined to send $V \in \mathcal{V}$ to $\Gamma^{d}\left(\operatorname{Hom}_{k}\left(k^{n}, V\right)\right)$, where $\Gamma^{d}(W)$ denotes the invariants under the symmetric group $\Sigma_{d}$ of the $d$-fold tensor power $W^{\otimes d}$ of the vector space $W$. Then $H_{o m}\left(\Gamma_{\mathcal{P}}^{d, n}, F\right)=$ $F\left(k^{n}\right)$ for any $F \in \mathcal{P}_{d}$, so that $\Gamma^{d, n}$ is projective. Furthermore, as shown in [F-S], $2.10, \Gamma^{d, n}$ is a projective generator of $\mathcal{P}_{d}$ provided that $n \geq d$. A precisely parallel argument shows that

$$
\operatorname{Hom}_{\mathcal{P}(2)}\left(\Gamma^{d, n} \odot \Gamma^{d^{\prime}, n^{\prime}}, T\right)=T\left(k^{n}, k^{n^{\prime}}\right)
$$

for any $T \in \mathcal{P}(2)$, so that each $\Gamma^{d, n} \odot \Gamma^{d^{\prime}, n^{\prime}}$ is projective; moreover, if $n \geq d, n^{\prime} \geq d^{\prime}$, then $\Gamma^{d, n} \odot \Gamma^{d^{\prime}, n^{\prime}}$ is a projective generator for $\mathcal{P}(2)_{d, d^{\prime}}$.

If $F=\Gamma^{d, n}$ and $F^{\prime}=\Gamma^{d^{\prime}, n^{\prime}}$, then the natural map

$$
\begin{aligned}
G\left(k^{n}\right) \otimes G\left(k^{n^{\prime}}\right)=\operatorname{Hom}_{\mathcal{P}}(F, G) \otimes & \operatorname{Hom}_{\mathcal{P}}\left(F^{\prime}, G^{\prime}\right) \\
& \rightarrow \operatorname{Hom}_{\mathcal{P}(2)}\left(F \odot F^{\prime}, G \odot G^{\prime}\right)=G\left(k^{n}\right) \otimes G^{\prime}\left(k^{n^{\prime}}\right)
\end{aligned}
$$

is the identity. Similarly, if $F, F^{\prime}$ are sums of such projective objects, then this map is an isomorphism. More generally, we choose projective resolutions $P_{\bullet} \rightarrow F, P_{\bullet}^{\prime} \rightarrow$ 
$F^{\prime}$ consisting of terms which are sums of such $\Gamma^{d, n}$. Then $P_{\bullet} \odot P_{\bullet}^{\prime} \rightarrow F \odot F^{\prime}$ is a projective resolution in $\mathcal{P}(2)$, so that the preceding special case together with the Künneth isomorphism implies that

$$
\begin{aligned}
\operatorname{Ext}_{\mathcal{P}}^{*}(F, G) \otimes \operatorname{Ext}_{\mathcal{P}}^{*}\left(G^{\prime}, G^{\prime}\right) \simeq H^{*}\left(\operatorname{Hom}_{\mathcal{P}}\left(P_{\bullet}, G\right) \otimes \operatorname{Hom}_{\mathcal{P}}\left(P_{\bullet}^{\prime}, G^{\prime}\right)\right) \\
\rightarrow H^{*}\left(\operatorname{Hom}_{\mathcal{P}(2)}\left(P \bullet \odot P_{\bullet}^{\prime}, G \odot G^{\prime}\right)\right) \simeq \operatorname{Ext}_{\mathcal{P}(2)}^{*}\left(F \odot F^{\prime}, G \odot G^{\prime}\right)
\end{aligned}
$$

is also an isomorphism.

Using the isomorphism of Proposition 3.6, we can now define a natural comultiplication on $E_{r}$ which provides it with the structure of a Hopf algebra.

Proposition 3.7. The natural map induced by the exact functors $\odot$ and $\Delta$ (and the natural isomorphism $\Delta I^{(r)}=I^{(r)} \odot I^{(r)}$ )

$$
\begin{aligned}
\nabla=\left(\odot^{*}\right)^{-1} \circ \Delta^{*}: \operatorname{Ext}_{\mathcal{P}}^{*}\left(I^{(r)}, I^{(r)}\right) \rightarrow & \operatorname{Ext}_{\mathcal{P}(2)}^{*}\left(I^{(r)} \odot I^{(r)}, I^{(r)} \odot I^{(r)}\right) \\
& \stackrel{\sim}{\longrightarrow} \operatorname{Ext}_{\mathcal{P}}^{*}\left(I^{(r)}, I^{(r)}\right) \otimes \operatorname{Ext}_{\mathcal{P}}^{*}\left(I^{(r)}, I^{(r)}\right)
\end{aligned}
$$

is a k-algebra homomorphism which provides $E_{r}(k)=\operatorname{Ext}_{\mathcal{P}}^{*}\left(I^{(r)}, I^{(r)}\right)$ with the structure of a Hopf algebra (with counit) over $k$. Furthermore, the map $E_{r-1}(k) \rightarrow$ $E_{r}(k)$ determined by Frobenius twist is an injective map of Hopf algebras. Finally the extension of scalars isomorphism $E_{r} \otimes_{\mathbb{F}_{p}} k=E_{r}(k)$ is compatible with the Hopf algebra structure.

Proof. The isomorphism $\odot^{*}$ of Proposition 3.6 is an isomorphism of algebras when $F=G, F^{\prime}=G^{\prime}$ provided that the product structure in $\operatorname{Ext}_{\mathcal{P}}^{*}(F, F) \otimes \operatorname{Ext}_{\mathcal{P}}^{*}\left(F^{\prime}, F^{\prime}\right)$ is defined using the graded tensor product (i.e., $\left(a \otimes a^{\prime}\right)\left(b \otimes b^{\prime}\right)=-1^{\operatorname{deg} a^{\prime} \operatorname{deg} b} a b \otimes a^{\prime} b^{\prime}$ for $a, a^{\prime}, b, b^{\prime}$ homogeneous elements). Since $E_{r}(k)$ is concentrated in even degrees, $\nabla$ is a $k$-algebra homomorphism.

Coassociativity of $\nabla$ is seen by identifying both functors $(\Delta, 1) \circ \Delta,(1, \Delta) \circ \Delta$ : $\mathcal{P} \rightarrow \mathcal{P}(3)$ with the functor sending $T$ to $\tau(T) \in \mathcal{P}(3)$ defined by $\tau(T)\left(V_{1}, V_{2}, V_{3}\right)=$ $T\left(V_{1} \otimes V_{2} \otimes V_{3}\right)$, where $\mathcal{P}(3)$ is the evident category of polynomial trifunctors.

The counit $\epsilon: E_{r}(k) \rightarrow k$ is defined to be the projection onto degree 0 :

$$
\epsilon: E_{r}(k) \rightarrow \operatorname{Hom}_{\mathcal{P}}\left(I^{(r)}, I^{(r)}\right)=\operatorname{Hom}_{k}\left(I^{(r)}(k), I^{(r)}(k)\right)=k .
$$

Let $\psi_{L}, \psi_{R}: \mathcal{P}(2) \rightarrow \mathcal{P}$ be the exact functors defined by

$$
\psi_{L}(T)(V)=T(k, V), \psi_{R}(T)(V)=T(V, k) .
$$

Then

$$
(\epsilon \otimes 1) \circ \nabla=\psi_{L}^{*} \circ \Delta^{*},(1 \otimes \epsilon) \circ \nabla=\psi_{R}^{*} \circ \Delta^{*}: E_{r}(k) \rightarrow E_{r}(k) .
$$

On the other hand, $\psi_{L} \circ \Delta=1=\psi_{R} \circ \Delta: \mathcal{P} \rightarrow \mathcal{P}$, so that $\epsilon$ is a counit for $\nabla$.

The fact that Frobenius twist determines a homomorphism (injective by Theorem 3.1) of Hopf algebras follows immediately from the observation that if we define $(-)^{(1)}: \mathcal{P}(2) \rightarrow \mathcal{P}(2)$ by setting $T^{(1)}(V, W)=T\left(V^{(1)}, W^{(1)}\right)$, then $\odot$ and $\Delta$ commute with Frobenius twists.

Compatibility of the extension of scalars isomorphism with comultiplication is obvious.

We introduce structure constants $\lambda_{i, j} \in \mathbb{F}_{p}$ for $E_{r}$ defined by the equation

$$
\nabla\left(e_{r}(\ell)\right)=\sum_{i+j=\ell} \lambda_{i, j} e_{r}(i) \otimes e_{r}(j) \in E_{r} \otimes E_{r} .
$$


Since the Hopf algebra map given by Frobenius twist, $E_{r-1} \rightarrow E_{r}$, sends $e_{r-1}(j)$ to $e_{r}(j)$ whenever $j<p^{r-1}, \lambda_{i, j}$ is well defined independent of $r$ (provided that $\left.i+j<p^{r}\right)$. In Theorem 4.6, we show that each of these structure constants $\lambda_{i, j}$ is equal to 1 .

Proposition 3.8. Let $G, G^{\prime}$ be affine group schemes over a field $k$. Let further $\rho$ : $G \rightarrow G L(V), \rho^{\prime}: G^{\prime} \rightarrow G L\left(V^{\prime}\right)$ be two finite-dimensional rational representations. Then we have the following formula relating characteristic classes:

$$
\begin{aligned}
e_{r}(\ell)\left(G \times G^{\prime}, V \otimes V^{\prime}\right) & =\sum_{i+j=\ell} \lambda_{i, j} e_{r}(i)(G, V) \otimes e_{r}(j)\left(G^{\prime}, V^{\prime}\right) \\
& \in H^{2 \ell}\left(G \times G^{\prime}, \operatorname{End}\left(V \otimes V^{\prime}\right)^{(r)}\right) \\
& =\bigoplus_{i+j=2 \ell} H^{i}\left(G, \operatorname{End}(V)^{(r)}\right) \otimes H^{j}\left(G^{\prime}, \operatorname{End}\left(V^{\prime}\right)^{(r)}\right) .
\end{aligned}
$$

Proof. Equality (3.8.1) is a consequence of the compatibility of $\odot^{*}$ and the Künneth isomorphism in group cohomology, in the sense of the commutativity of the following diagram:

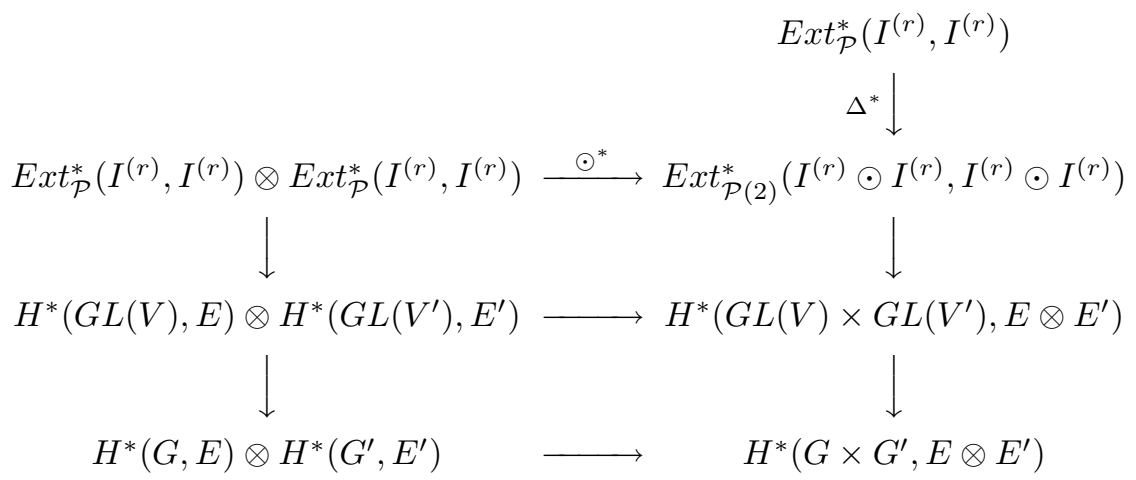

where $E=\operatorname{End}\left(V^{(r)}\right), E^{\prime}=\operatorname{End}\left(V^{\prime}(r)\right)$. This commutativity is a consequence of the fact that each of the three horizontal isomorphisms arise from the Künneth theorem for the cohomology of the tensor product of chain complexes.

\section{§4. Explicit COMPUTATIONS}

In this section, we explicitly determine the characteristic classes $e_{r}\left(\left(\mathbb{G}_{a(r)}\right)_{A}, V_{\underline{\alpha}}\right)$ associated to a 1-parameter subgroup $\exp _{\underline{\alpha}}: \mathbb{G}_{a(r)} \otimes_{\mathbb{F}_{p}} A \rightarrow G L_{n} \otimes_{\mathbb{F}_{p}} A$. This is done in several stages; for the special case $r=1$, our convention establishes an agreeable answer; for the special case $r \leq 2$, a weight argument enables us to extend this answer provided that our 1-parameter subgroup is given by a single $p$-nilpotent matrix. This is sufficient for us to determine explicitly the Hopf algebra structure on $E_{r}$; we use the fact that the product $\exp _{\alpha} \cdot \exp _{\beta}$ can be rewritten as $\exp _{\alpha+\beta} \cdot \exp _{\zeta}^{(1)}$ (cf. (4.6.0)). Finally, the knowledge of this Hopf algebra structure facilitates an induction argument to complete the determination.

Let $A$ be a commutative $\mathbb{F}_{p}$-algebra and let $\underline{\alpha}=\left(\alpha_{0}, \ldots, \alpha_{l-1}\right) \in M_{n}(A)^{\times l}$ be an $l$-tuple of pairwise commuting $p$-nilpotent $n \times n$ matrices. For every $r>0$, this data defines a representation

$$
\mathbb{G}_{a(r)}^{\times l} \otimes_{\mathbb{F}_{p}} A \rightarrow G L_{n} \otimes_{\mathbb{F}_{p}} A:\left(t_{0}, \ldots, t_{l-1}\right) \mapsto \exp _{\alpha_{0}}\left(t_{0}\right) \cdot \ldots \cdot \exp _{\alpha_{l-1}}\left(t_{l-1}\right) .
$$


This representation makes $A^{n}$ into a rational $\left(\mathbb{G}_{a(r)}^{\times l}\right)_{A}$-module, which we denote $W_{\underline{\alpha}}$. Furthermore, for every $r \geq l$ we may consider the corresponding representation

$$
\exp _{\underline{\alpha}}: \mathbb{G}_{a(r)} \otimes_{\mathbb{F}_{p}} A \rightarrow G L_{n} \otimes_{\mathbb{F}_{p}} A: t \mapsto \exp _{\alpha_{0}}(t) \cdot \ldots \cdot \exp _{\alpha_{l-1}}\left(t^{p^{l-1}}\right) .
$$

We denote by $V_{\underline{\alpha}}$ the free $A$-module $A^{n}$ made into a rational $\mathbb{G}_{a(r)} \otimes_{\mathbb{F}_{p}} A$-module via this representation. Clearly $V_{\underline{\alpha}}$ is a pull-back of the rational module $W_{\underline{\alpha}}$ under the homomorphism $\mathbb{G}_{a(r)} \stackrel{1 \times F \times \ldots \times F^{l-1}}{\longrightarrow} \mathbb{G}_{a(r)}^{\times l}$.

Throughout this section $k$ denotes a field of characteristic $p>0$ and $A$ denotes an arbitrary commutative $\mathbb{F}_{p}$-algebra.

Proposition 4.1. Consider the representation $\exp _{\alpha}: \mathbb{G}_{a(1)} \rightarrow G L_{n}$ determined by the $p$-nilpotent matrix $\alpha \in M_{n}(k)$ and let $V_{\alpha}$ denote the associated rational $\mathbb{G}_{a(1)^{-}}$ module. Then

$$
\begin{aligned}
& e_{r}\left(\mathbb{G}_{a(1)}, V_{\alpha}\right)=x_{1}^{p^{r-1}} \otimes \alpha^{(r)} \in H^{2 p^{r-1}}\left(\mathbb{G}_{a(1)}, M_{n}(k)^{(r)}\right) \\
& \quad=H^{2 p^{r-1}}\left(\mathbb{G}_{a(1)}, k\right) \otimes_{k} M_{n}(k)^{(r)}=H^{2 p^{r-1}}\left(\mathbb{G}_{a(1)}, k\right) \otimes_{k} M_{n}(k),
\end{aligned}
$$

where $x_{1} \in H^{2}\left(\mathbb{G}_{a(1)}, k\right)$ is the canonical generator of $H^{*}\left(\mathbb{G}_{a(1)}, k\right)$ (see Theorem 1.13).

Proof. The construction of the May spectral sequence implies that the element $x_{1} \in$ $H^{2}\left(\mathbb{G}_{a(1)}, k\right)$ coincides with the image of $t^{(1)} \in g_{a}^{(1) \#}$ under the edge homomorphism of the May spectral sequence, where $k\left[\mathbb{G}_{a(1)}\right]=k[T] / T^{p}$ and $t$ denotes the class of $T$ in $\operatorname{Lie}\left(\mathbb{G}_{a}\right)^{\#}=(T) /\left(T^{2}\right)$. Let $\left\{E^{i, j}\right\}$ denote the basis of $M_{n}(k)^{\#}$ dual to the usual basis $\left\{E_{i, j}\right\}$ of $M_{n}(k)$. The naturality of the May spectral sequence implies the commutativity of the following diagram:

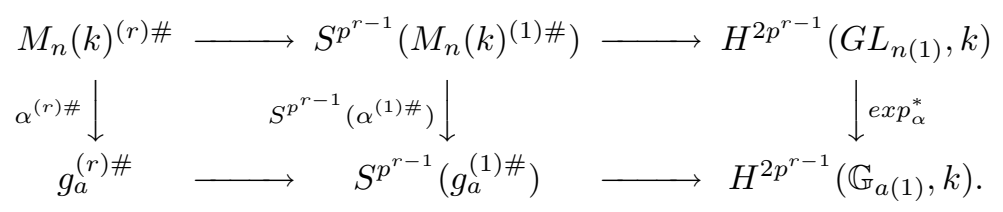

The top row coincides (according to Convention 3.4) with the map $g l_{n}^{(r) \#} \rightarrow$ $H^{2 p^{r-1}}\left(G L_{n(1)}, k\right)$ corresponding to $e_{r}\left(G L_{n(1)}, k^{n}\right)$. The bottom row sends $t^{(r)}$ to $x_{1}^{p^{r-1}}$ by the preceding remark. Thus the map $M_{n}(k)^{(r) \#} \rightarrow H^{2 p^{r-1}}\left(\mathbb{G}_{a(1)}, k\right)$ corresponding to $e_{r}\left(\mathbb{G}_{a(1)}, V_{\alpha}\right)$ sends $\left(E^{i, j}\right)^{(r)}$ to $\left(\alpha_{i j}\right)^{p^{r}} x_{1}^{p^{r-1}}$.

The following lemma establishes the effect of Frobenius on our characteristic classes.

Lemma 4.2. Let $F^{*}: H^{*}\left(\mathbb{G}_{a(\ell-1)}, k\right) \rightarrow H^{*}\left(\mathbb{G}_{a(\ell)}, k\right)$ denote the map induced by Frobenius. For any $0<\ell \leq r$ and any $\ell$-tuple $\underline{\alpha}=\left(\alpha_{0}, \ldots, \alpha_{\ell-1}\right) \in M_{n}(k)^{\times \ell}$ of commuting p-nilpotent matrices,

(a) $e_{r}(j)\left(\mathbb{G}_{a(\ell)}, V_{\left(0, \alpha_{1}, \ldots \alpha_{\ell-1}\right)}\right)=F^{*}\left(e_{r}(j)\left(\mathbb{G}_{a(\ell-1)}, V_{\left(\alpha_{1}, \ldots, \alpha_{\ell-1}\right)}\right)\right)$.

(b) If $j<p^{r-1}$, then

$$
e_{r}(j)\left(\mathbb{G}_{a(\ell)}, V_{\left(\alpha_{0}, \ldots, \alpha_{\ell-1}\right)}\right)=F^{*}\left(e_{r-1}(j)\left(\mathbb{G}_{a(\ell-1)}, V_{\left(\alpha_{0}^{(1)}, \ldots, \alpha_{\ell-2}^{(1)}\right)}\right)\right) .
$$

Proof. Assertion (a) follows from the definition of $e_{r}(j)(G, V)$ and the fact that $\exp _{\left(0, \alpha_{1}, \ldots, \alpha_{\ell-1}\right)}=\exp _{\left(\alpha_{1}, \ldots, \alpha_{\ell-1}\right)} \circ F: \mathbb{G}_{a(\ell)} \rightarrow G L_{n}$. To prove assertion (b), it 
suffices to establish the commutativity of the following diagram:

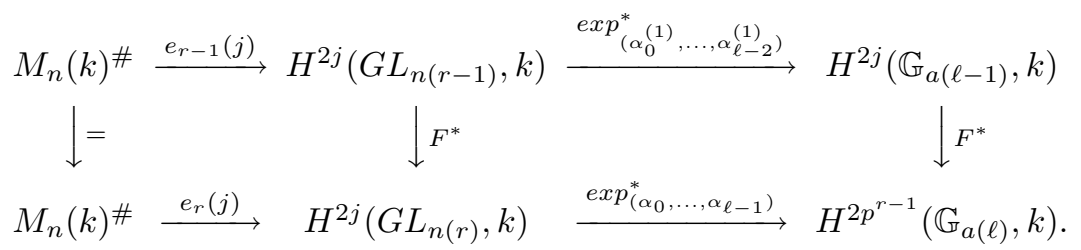

Commutativity of the right square follows from the obvious relation $\exp \left(\alpha_{0}^{(1)}, \ldots, \alpha_{\ell-2}^{(1)}\right)^{\circ}$ $F=F \circ \exp _{\left(\alpha_{0}, \ldots, \alpha_{\ell-1}\right)}: \mathbb{G}_{a(\ell)} \rightarrow G L_{n}$. To check the commutativity of the left square note first of all that $e_{r}(j)=e_{r-1}(j)^{(1)}\left(\right.$ for $\left.j<p^{r-1}\right)$. Let

$$
0 \rightarrow I^{(r-1)} \stackrel{d}{\rightarrow} T_{1} \stackrel{d}{\rightarrow} \ldots \stackrel{d}{\rightarrow} T_{2 j} \stackrel{d}{\rightarrow} I^{(r-1)} \rightarrow 0
$$

be an extension of polynomial functors representing $e_{r-1}(j)$. The extension class $e_{r}(j)=e_{r-1}(j)^{(1)}$ is represented by the extension

$$
0 \rightarrow I^{(r)} \stackrel{d^{(1)}}{\longrightarrow} T_{1}^{(1)} \stackrel{d^{(1)}}{\longrightarrow} \ldots \stackrel{d^{(1)}}{\longrightarrow} T_{2 j}^{(1)} \stackrel{d^{(1)}}{\longrightarrow} I^{(r)} \rightarrow 0
$$

(where $T^{(1)}=T \circ I^{(1)}$ for any $T \in \mathcal{P}$ ) and hence $e_{r}(j)\left(G L_{n(r)}, k^{n}\right)$ is represented by the extension of rational $G L_{n(r)}$-modules

$0 \rightarrow\left(k^{n}\right)^{(r)} \stackrel{d\left(k^{n(1)}\right)}{\longrightarrow} T_{1}\left(k^{n(1)}\right) \stackrel{d\left(k^{n(1)}\right)}{\longrightarrow} \ldots \stackrel{d\left(k^{n(1)}\right)}{\longrightarrow} T_{2 j}\left(k^{n(1)}\right) \stackrel{d\left(k^{n(1)}\right)}{\longrightarrow}\left(k^{n}\right)^{(r)} \rightarrow 0$.

For any polynomial functor $T \in \mathcal{P}$, the vector space $T\left(k^{n(1)}\right)$ may be identified with $T\left(k^{n}\right)$ and the representation of $G L_{n}$ in $T\left(k^{n(1)}\right)$ coincides with the composition of the representation of $G L_{n}$ in $T\left(k^{n}\right)$ and the Frobenius endomorphism of $G L_{n}$. This shows that the above extension of rational $G L_{n(r)}$-modules is the pull-back via the Frobenius homomorphism $G L_{n(r)} \stackrel{F}{\longrightarrow} G L_{n(r-1)}$ of the extension representing $e_{r-1}(j)\left(G L_{n(r-1)}, k^{n}\right)$.

The following lemma reduces our computations to the special case $A=\mathbb{F}_{p}$ and the very special representation given as the external tensor product of $p \times p$ Jordan blocks. This is particularly useful in that Proposition 3.8 applies to analyze $e_{r}\left(\mathbb{G}_{a(\ell)}^{\times i} \otimes_{\mathbb{F}_{p}} A, W_{\left(\alpha_{1}, \ldots, \alpha_{i}\right)}\right)$.

Lemma 4.3. Let $P\left(X_{1}, \ldots, X_{i}\right)$ be a polynomial in $i$ variables with coefficients in the cohomology ring $H^{e v}\left(\mathbb{G}_{a(\ell)}^{\times i}, \mathbb{F}_{p}\right)$. Let further $r \geq \ell$ be an integer. Assume that the formula

$$
e_{r}\left(\mathbb{G}_{a(\ell)}^{\times i} \otimes_{\mathbb{F}_{p}} A, W_{\left(\alpha_{1}, \ldots, \alpha_{i}\right)}\right)=P\left(\alpha_{1}^{(r)}, \ldots, \alpha_{i}^{(r)}\right)
$$

holds in the following special case: $A=\mathbb{F}_{p}$;

$$
\alpha_{1}=\alpha \otimes 1 \otimes \ldots \otimes 1, \ldots, \alpha_{i}=1 \otimes 1 \otimes \ldots \otimes \alpha,
$$

where $\alpha$ is a $p \times p$ Jordan block. Then the above formula holds for any $A$ and any $i$-tuple of commuting p-nilpotent matrices.

Proof. To give an $i$-tuple of commuting $p$-nilpotent matrices $\alpha_{1}, \ldots, \alpha_{i} \in M_{n}(A)$ is equivalent to making $A^{n}$ into a module over the $A$-algebra

$$
B=A\left[Y_{1}, \ldots, Y_{i}\right] /\left(Y_{1}^{p}, \ldots, Y_{i}^{p}\right)=A\left[Y_{1}\right] / Y_{1}^{p} \otimes_{A} \ldots \otimes_{A} A\left[Y_{i}\right] / Y_{i}^{p} .
$$


The set of matrices corresponding to the free rank one $B$-module $B$ is precisely the above special set $\left\{\alpha_{1}, \ldots, \alpha_{i}\right\}$. Thus our condition and Proposition 3.2(c) show that the formula in question holds for a free $B$-module of rank one. Proposition 3.2 (b) implies that the formula holds for any free $B$-module of finite rank. Finally we observe that any finitely generated $B$-module is a quotient of a free $B$-module of finite rank. So to end the proof, it suffices to establish the following fact.

4.3.2. Let $\alpha_{1}, \ldots, \alpha_{i} \in M_{n}(A)$ and $\beta_{1}, \ldots, \beta_{i} \in M_{m}(A)$ be two $i$-tuples of commuting p-nilpotent matrices. Assume further that the equality (4.3.1) holds for $\beta$ and that there exists a surjective A-linear map $\phi: A^{m} \rightarrow A^{n}$ such that $\alpha_{j} \phi=\phi \beta_{j}$ for all $j=1, \ldots, i$. Then the formula (4.3.1) holds for $\alpha$ as well.

Proof. To simplify the notation, denote the cohomology ring $H^{e v}\left(\mathbb{G}_{a(\ell)}^{\times i}, \mathbb{F}_{p}\right)$ by $H$. Since $\phi$ is surjective, the induced map

$$
\operatorname{End}_{A}\left(\left(A^{n}\right)^{(r)}\right) \otimes_{\mathbb{F}_{p}} H \stackrel{\left(\phi^{(r)}\right)^{*} \otimes 1}{\longrightarrow} \operatorname{Hom}_{A}\left(\left(A^{m}\right)^{(r)},\left(A^{n}\right)^{(r)}\right) \otimes_{\mathbb{F}_{p}} H
$$

is injective. Thus it suffices to check that both sides of the formula (4.3.1) give the same result being composed with $\phi^{(r)}$ on the right. However according to Proposition 3.2(a) we have:

$$
\begin{aligned}
e_{r}\left(\mathbb{G}_{a(\ell)}^{\times i} \otimes_{\mathbb{F}_{p}} A, W_{\underline{\alpha}}\right) \cdot \phi^{(r)} & =\phi^{(r)} \cdot e_{r}\left(\mathbb{G}_{a(\ell)}^{\times i} \otimes_{\mathbb{F}_{p}} A, W_{\underline{\beta}}\right)=\phi^{(r)} \cdot P\left(\beta_{1}^{(r)}, \ldots, \beta_{i}^{(r)}\right) \\
& =P\left(\alpha_{1}^{(r)}, \ldots \alpha_{i}^{(r)}\right) \cdot \phi^{(r)} .
\end{aligned}
$$

The next proposition is the extension of Proposition 4.1 to $\mathbb{G}_{a(2)}$.

Proposition 4.4. Consider the representation $\exp _{\alpha}: \mathbb{G}_{a(2)} \rightarrow G L_{n}$ determined by the p-nilpotent matrix $\alpha \in M_{n}(k)$, and let $V_{\alpha}$ denote the associated rational $\mathbb{G}_{a(2)}$-module. Then for any $r \geq 2$

$$
e_{r}\left(\mathbb{G}_{a(2)}, V_{\alpha}\right)=x_{1}^{p^{r-1}} \otimes \alpha^{(r)} \in H^{2 p^{r-1}}\left(\mathbb{G}_{a(2)}, k\right) \otimes_{k} M_{n}(k)^{(r)} .
$$

Proof. By Lemma 4.3 it suffices to consider $\alpha$ to be a $p \times p$ Jordan block. We may also assume (extending the scalars if necessary) that the field $k$ is infinite.

Let $T \subset G L_{p}$ be the rank-1 split torus whose $k$-rational points are matrices of the form $d_{s} \equiv \operatorname{diag}\left(1, s, \ldots, s^{p-1}\right) \quad\left(s \in k^{*}\right)$. So defined, $T$ stabilizes $\exp _{\alpha}$ since $d_{s} \alpha d_{s}^{-1}=s^{-1} \alpha$ and hence $d_{s} \exp _{\alpha} d_{s}^{-1}=\exp _{\alpha} \circ s^{-1}$. Since the cohomology class $e_{r} \in$ $H^{2 p^{r-1}}\left(G L_{p}, M_{p}(k)^{(r)}\right)$ is invariant under the action of $G L_{p}$ by conjugation on $G L_{p}$ and $M_{p}(k)$, we conclude that the class $e_{r}\left(\mathbb{G}_{a(2)}, V_{\alpha}\right)$ is invariant under the action of $T$ (where $d_{s}$ acts on $M_{p}(k)^{(r)}$ by conjugation and on $\mathbb{G}_{a(2)}$ by multiplication by $\left.s^{-1}\right)$.

We write

$$
\begin{aligned}
e_{r}\left(\mathbb{G}_{a(2)}, V_{\alpha}\right) & =\sum_{i, j=1}^{p} x_{i, j} \otimes E_{i, j}^{(r)} \in H^{2 p^{r-1}}\left(\mathbb{G}_{a(2)}, M_{p}(k)^{(r)}\right) \\
& =H^{2 p^{r-1}}\left(\mathbb{G}_{a(2)}, k\right) \otimes_{k} M_{p}(k)^{(r)}
\end{aligned}
$$

for some elements $x_{i, j} \in H^{2 p^{r-1}}\left(\mathbb{G}_{a(2)}, k\right)$. As $d_{s} E_{i, j}^{(r)} d_{s}^{-1}=s^{p^{r}(i-j)} E_{i, j}^{(r)}$, we must have $\left(d_{s^{-1}}\right)^{*}\left(x_{i, j}\right)=s^{-p^{r}(i-j)} x_{i, j}=s^{p^{r}(j-i)} x_{i, j}$ for all $i, j$. In other words, $x_{i, j} \in$ 
$H^{2 p^{r-1}}\left(\mathbb{G}_{a(2)}, k\right)$ must have weight $p^{r}(j-i)$ with respect to the standard action of $\mathbb{G}_{m}$ on $\mathbb{G}_{a(2)}$.

Sublemma 4.4.1. Let $r \geq \ell>0$ be a pair of integers. The only monomial of weight $p^{r}$ in $H^{2 p^{r-1}}\left(\mathbb{G}_{a(\ell)}, k\right)$ (with respect to the standard action of $\mathbb{G}_{m}$ on $\mathbb{G}_{a(\ell)}$ ) is $x_{1}^{p^{r-1}}$. If $\ell \leq 2$, then there are no monomials in $H^{2 p^{r-1}}\left(\mathbb{G}_{a(\ell)}, k\right)$ of weight $p^{r} m$ with $1<m<p$.

Proof. Consider an arbitrary monomial $x=x_{1}^{n_{1}} \cdot \ldots \cdot x_{\ell}^{n_{\ell}} \cdot \lambda_{1}^{\epsilon_{1}} \cdot \ldots \cdot \lambda_{\ell}^{\epsilon_{\ell}}\left(0 \leq \epsilon_{j} \leq 1\right)$. Its degree equals $2\left(n_{1}+\ldots+n_{\ell}\right)+\left(\epsilon_{1}+\ldots+\epsilon_{\ell}\right)$ and its weight equals $n_{1} p+\ldots+$ $n_{\ell} p^{\ell}+\epsilon_{1}+\epsilon_{2} p+\ldots+\epsilon_{\ell} p^{\ell-1}$. Since $w(x)$ has to be divisible by $p$ we conclude that $\epsilon_{1}=0$. Now it's straightforward to check that $w(x) \geq \frac{p}{2} \operatorname{deg}(x)$ and the equality holds only if $\epsilon_{2}=\ldots=\epsilon_{\ell}=n_{2}=\ldots=n_{\ell}=0$. This proves the first statement. The second statement is trivial for $\ell=1$. Assume that $\ell=2$ and $x=x_{1}^{n_{1}} x_{2}^{n_{2}} \lambda_{1}^{\epsilon_{1}} \lambda_{2}^{\epsilon_{2}}$ has degree $2 p^{r-1}$ and weight $p^{r-1} m$ with $1<m<p$. As before $\epsilon_{1}=0$ and since $\operatorname{deg}(x)$ has to be even we conclude that $\epsilon_{2}=0$. Thus we have a system of equations $n_{1}+n_{2}=p^{r-1}, n_{1} p+n_{2} p^{2}=p^{r} m$ from which we conclude that $n_{2}(p-1)=p^{r-1}(m-1)$ and thus $m-1 \equiv 0 \bmod p-1$, which gives us a contradiction.

Sublemma 4.4.1 shows that $x_{i, j}=0$ unless $j=i+1$ and $x_{i, i+1}=c_{i} x_{1}^{p^{r-1}}$ for some $c_{i} \in k$. Restricting the class $e_{r}\left(\mathbb{G}_{a(2)}, V_{\alpha}\right)$ to $\mathbb{G}_{a(1)}$ and applying Proposition 4.1 we conclude that each $c_{i}=1$ and so

$$
e_{r}\left(\mathbb{G}_{a(2)}, V_{\alpha}\right)=\sum_{i=1}^{p-1} x_{1}^{p^{r-1}} \otimes E_{i, i+1}^{(r)}=x_{1}^{p^{r-1}} \otimes \alpha^{(r)} .
$$

We can now apply Lemma 4.3 to get an almost complete understanding of characteristic classes for $\mathbb{G}_{a(2)}$.

Corollary 4.5. (a) Let $\alpha \in M_{n}(k)$ be a p-nilpotent matrix. Then for any $r \geq 2$ and any $0 \leq j<p^{r}$

$$
e_{r}(j)\left(\mathbb{G}_{a(2)}, V_{\alpha}\right)=\frac{x_{r}^{j_{0}} \cdot \ldots \cdot x_{1}^{j_{r-1} p^{r-1}}}{j_{0} ! \cdot \ldots \cdot j_{r-1} !} \otimes\left(\alpha^{(r)}\right)^{s(j)}
$$

where $j=j_{0}+j_{1} p+\ldots+j_{r-1} p^{r-1} \quad\left(0 \leq j_{i}<p\right)$ is the $p$-adic expansion of $j$ and $s(j)=j_{0}+\ldots+j_{r-1}$ is the sum of p-adic digits of $j$. In particular, $e_{r}(j)\left(\mathbb{G}_{a(2)}, V_{\alpha}\right)=$ 0 unless $j \equiv 0 \bmod p^{r-2}$ since $x_{i}=0 \in H^{*}\left(G_{a(2)}, k\right)$ whenever $i>2$.

(b) Let $\alpha, \beta \in M_{n}(k)$ be a pair of commuting p-nilpotent matrices. Then for any $r \geq 2$

$$
\begin{aligned}
e_{r}\left(\mathbb{G}_{a(2)}^{\times 2}, W_{(\alpha, \beta)}\right)= & \left(x_{1}^{p^{r-1}} \otimes 1\right) \otimes \alpha^{(r)}+\left(1 \otimes x_{1}^{p^{r-1}}\right) \otimes \beta^{(r)} \\
& +\sum_{i+j=p, 0 \neq i \neq p} \lambda\left(i p^{r-2}, j p^{r-2}\right) \frac{x_{2}^{i p^{r-2}} \otimes x_{2}^{j p^{r-2}}}{i ! \cdot j !} \otimes\left(\alpha^{(r)}\right)^{i}\left(\beta^{(r)}\right)^{j}
\end{aligned}
$$

Proof. According to the definition of $e_{r}(j)$, we have

$$
e_{r}(j)\left(\mathbb{G}_{a(2)}, V_{\alpha}\right)=\frac{e_{1}^{(r-1)}\left(\mathbb{G}_{a(2)}, V_{\alpha}\right)^{j_{0}} \cdot \ldots \cdot e_{r}\left(\mathbb{G}_{a(2)}, V_{\alpha}\right)^{j_{r-1}}}{j_{0} ! \cdot \ldots \cdot j_{r-1} !} .
$$


Proposition 4.4, Proposition 4.1, and Lemma 4.2(b) show that

$$
e_{i}^{(r-i)}\left(\mathbb{G}_{a(2)}, V_{\alpha}\right)=x_{r+1-i}^{p^{i-1}} \otimes \alpha^{(r)}(=0 \text { if } i<r-1) .
$$

This proves the first part of our statement. Lemma 4.3 shows that in proving the second part we may assume that $\alpha=\gamma \otimes 1, \beta=1 \otimes \gamma$, where $\gamma$ is the $p \times p$ Jordan block. In this case $W_{(\alpha, \beta)}=V_{\gamma} \otimes V_{\gamma}$, so that our statement follows from (a) and Proposition 3.8 .

If $\alpha$ and $\beta$ are two commuting $p$-nilpotent matrices in $M_{n}(k)$, then for any $s$ we have the equality

$$
\exp (s \cdot \alpha) \cdot \exp (s \cdot \beta)=\exp (s \cdot(\alpha+\beta)) \cdot \exp \left(s^{p} \cdot \xi\right)
$$

where $\xi=\sum_{i+j=p, 0 \neq i \neq p} \frac{\alpha^{i} \beta^{j}}{i ! j !}$. Thus, we conclude

$$
\exp _{\alpha} \cdot \exp _{\beta}=\exp _{\alpha+\beta} \cdot \exp _{\xi}^{(1)}: \mathbb{G}_{a(r)} \rightarrow G L_{n}
$$

This simple observation in conjunction with Corollary 4.5 enables us to determine the structure constants $\lambda_{i, j}$ of the Hopf algebra $E_{r}$.

Theorem 4.6. $E_{r}$ is isomorphic as a Hopf algebra to the dual $\left(\mathbb{F}_{p}[X] / X^{p^{r}}\right)^{\#}$ of the primitively generated truncated polynomial algebra $\mathbb{F}_{p}[X] / X^{p^{r}}$. Furthermore, the structure constants $\lambda_{i, j}$ of (3.8.0) all equal 1 ; in particular,

$$
\nabla\left(e_{r}\right)=\sum_{i+j=p^{r-1}} e_{r}(i) \otimes e_{r}(j) .
$$

Proof. Recall that $\left(\mathbb{F}_{p}[X] / X^{p^{r}}\right)^{\#}=\mathbb{F}_{p}\left[u_{0}, \ldots, u_{r-1}\right] /\left(u_{0}^{p}, \ldots, u_{r-1}^{p}\right)$ (see Corollary 1.4). Theorem 3.1 shows that sending $u_{i}$ to $e_{i+1}^{(r-i-1)}$ defines an isomorphism of algebras $\phi_{r}:\left(\mathbb{F}_{p}[X] / X^{p^{r}}\right)^{\#} \stackrel{\sim}{\longrightarrow} E_{r}$. This isomorphism takes $v_{j}$ (as in Corollary 1.4) to $e_{r}(j)$ for all $0 \leq j<p^{r}$. To show that $\phi_{r}$ is an isomorphism of Hopf algebras, it suffices (since $\nabla\left(v_{\ell}\right)=\sum_{i+j=\ell} v_{i} \otimes v_{j}$ ) to show that all structure constants $\lambda_{i, j}$ are equal to 1 . To prove this, we proceed by induction on $r$. For $r=0$ there's nothing to prove. Assume now that the statement is true for $r-1$ and consider the case of $E_{r}$. Our induction hypothesis and the fact that $\lambda_{i, j}$ is independent of $r$ (provided that $i+j<p^{r}$ ) show that $\lambda_{i, j}=1$ provided that $i+j<p^{r-1}$. Let $\alpha, \beta \in M_{n}\left(\mathbb{F}_{p}\right)$ be a pair of commuting $p$-nilpotent matrices. Consider the representation $\exp _{\alpha} \cdot \exp _{\beta}: \mathbb{G}_{a(2)} \rightarrow G L_{n}$. Denote by $U$ the vector space $\mathbb{F}_{p}^{n}$ made into a rational $\mathbb{G}_{a(2)}$-module via this representation. Note that $U$ is the pull-back of the representation $W_{(\alpha, \beta)}$ under the diagonal embedding $\Delta: \mathbb{G}_{a(2)} \rightarrow \mathbb{G}_{a(2)}^{\times 2}$. On the other hand, (4.6.0) shows that $U$ is a pull-back of the representation $W_{(\alpha+\beta, \xi)}$ under the homomorphism $\mathbb{G}_{a(2)} \stackrel{(1, F)}{\longrightarrow} \mathbb{G}_{a(2)}^{\times 2}$. These remarks and Corollary 4.5 give us two ways to compute $e_{r}\left(\mathbb{G}_{a(2)}, U\right)$. Comparing the results we get the following relation:

$$
\begin{aligned}
x_{1}^{p^{r-1}} & \otimes\left(\alpha^{(r)}+\beta^{(r)}\right)+x_{2}^{p^{r-1}} \otimes \sum_{i+j=p, 0 \neq i \neq p} \frac{\lambda\left(i p^{r-2}, j p^{r-2}\right)}{i ! \cdot j !} \cdot\left(\alpha^{(r)}\right)^{i}\left(\beta^{(r)}\right)^{j} \\
& =x_{1}^{p^{r-1}} \otimes(\alpha+\beta)^{(r)}+x_{2}^{p^{r-1}} \otimes \xi^{(r)} .
\end{aligned}
$$


Since $x_{2}^{p^{r-1}} \neq 0$, we conclude from the previous formula that

$$
\sum_{i+j=p, 0 \neq i \neq p} \frac{\lambda\left(i p^{r-2}, j p^{r-2}\right)-1}{i ! \cdot j !} \alpha^{i} \beta^{j}=0 .
$$

Taking $\alpha$ and $\beta$ to be multiplication by $x$ and $y$ in the $\operatorname{ring} B=\mathbb{F}_{p}[x, y] /\left(x^{p}, y^{p}\right.$ ) (in which case the monomials $\alpha^{i} \beta^{j}$ with $i, j<p$ are linearly independent over $\mathbb{F}_{p}$ ) we conclude immediately that all coefficients in (4.6.1) are zero, i.e. $\lambda\left(i p^{r-2}, j p^{r-2}\right)=1$ whenever $i+j=p, 0 \neq i \neq p$.

Next we show that $\lambda(i, j)=1$ whenever $i+j=p^{r-1}$. To do so, we note that coassociativity of $E_{r}$ gives the following relation on $\lambda$ :

$$
\lambda(a, b) \cdot \lambda(a+b, c)=\lambda(a, b+c) \cdot \lambda(b, c) \quad\left(0 \leq a, b, c ; a+b+c<p^{r}\right) .
$$

Assume first that $i \geq p^{r-2}$. Taking in the above relation $a=p^{r-2}, b=i-p^{r-2}, c=j$ and taking into account that $\lambda\left(p^{r-2},(p-1) p^{r-2}\right)=1$ and also $\lambda(a, b)=\lambda(b, c)=1$ since $a+b<p^{r-1}, b+c<p^{r-1}$, we conclude that $\lambda(i, j)=1$. If $i<p^{r-2}$, then $j \geq p^{r-2}$ and a similar argument works.

Consider the diagram

$$
\begin{array}{ccc}
\mathbb{F}_{p}\left[u_{0}, \ldots, u_{r-1}\right] /\left(u_{0}^{p}, \ldots, u_{r-1}^{p}\right) & \stackrel{\nabla}{\longrightarrow}\left(\mathbb{F}_{p}\left[u_{0}, \ldots u_{r-1}\right] /\left(u_{0}^{p}, \ldots, u_{r-1}^{p}\right)\right)^{\otimes 2} \\
\cong \downarrow \phi_{r} & & \cong \downarrow \phi_{r}^{\otimes 2} \\
E_{r} & \stackrel{\nabla}{\longrightarrow} & E_{r}^{\otimes 2} .
\end{array}
$$

The above computation shows that the two images of $u_{r-1}$ in $E_{r}^{\otimes 2}$ coincide. The same being true for $u_{0}, \ldots, u_{r-2}$ in view of the induction hypothesis we conclude that $\phi_{r}$ is an isomorphism of Hopf algebras and $\lambda_{i, j}=1$ for all $i, j$ such that $i+j<p^{r}$.

Using Theorem 4.6, we can now complete our determination of the characteristic classes $e_{r}(j)\left(\mathbb{G}_{a(\ell)}, V_{\alpha}\right)$ associated to a 1-parameter subgroup given by a single $p$ nilpotent matrix $\alpha$.

Theorem 4.7. For any $0<\ell \leq r$, any $0 \leq j<p^{r}$ and any p-nilpotent matrix $\alpha \in M_{n}(k)$,

$$
e_{r}(j)\left(\mathbb{G}_{a(\ell)}, V_{\alpha}\right)=\frac{x_{r}^{j_{0}} \cdots x_{1}^{p^{r-1} j_{r-1}}}{\left(j_{0} !\right) \cdots\left(j_{r-1} !\right)} \otimes\left(\alpha^{(r)}\right)^{s(j)}
$$

where $j=j_{0}+j_{1} p+\cdots+j_{r-1} p^{r-1}\left(0 \leq j_{i}<p\right)$ is the $p$-adic expansion of $j$ and where $s(j)=\sum j_{i}$ is the sum of $p$-adic digits of $j$.

Proof. The cases $\ell=1,2$ have been verified in Propositions 4.1 and 4.4. In dealing with the general case, we may assume, using induction on $\ell$, that the theorem holds for $\ell-1$. Assume first that $j<p^{r-1}$ (so that $j_{r-1}=0$ ). In this case induction and Lemma $4.2(\mathrm{~b})$ show

$$
\begin{aligned}
e_{r}(j) & \left(\mathbb{G}_{a(\ell)}, V_{\alpha}\right)=F^{*}\left(e_{r-1}(j)\left(\mathbb{G}_{a(\ell-1)}, V_{\alpha(1)}\right)\right) \\
& =F^{*}\left(\frac{x_{r-1}^{j_{0}} \cdot \ldots \cdot x_{1}^{j_{r-2}} p^{r-2}}{j_{0} ! \cdot \ldots \cdot j_{r-2} !}\right) \otimes\left(\alpha^{(r)}\right)^{s(j)}=\frac{x_{r}^{j_{0}} \cdot \ldots \cdot x_{1}^{j_{r-1} p^{r-1}}}{j_{0} ! \cdot \ldots \cdot j_{r-1} !} \otimes\left(\alpha^{(r)}\right)^{s(j)} .
\end{aligned}
$$

To settle the general case, it suffices now to prove the formula

$$
e_{r}\left(\mathbb{G}_{a(\ell)}, V_{\alpha}\right)=x_{1}^{p^{r-1}} \otimes \alpha^{(r)} .
$$


Moreover Lemma 4.3 shows that we may assume that $\alpha$ is a $p \times p$ Jordan block. As in the proof of Proposition 4.4, we may write

$$
e_{r}\left(\mathbb{G}_{a(\ell)}, V_{\alpha}\right)=\sum_{1 \leq i<j<p} x_{i, j} \otimes E_{i, j}^{(r)} \in H^{2 p^{r-1}}\left(\mathbb{G}_{a(\ell)}, k\right) \otimes_{k} M_{p}(k)^{(r)}
$$

for some $x_{i, j} \in H^{2 p^{r-1}}\left(\mathbb{G}_{a(\ell)}, \mathbb{F}_{p}\right)$ of weight $p^{r}(j-i)$ with respect to the standard action of $\mathbb{G}_{m}$ on $\mathbb{G}_{a(\ell)}$. The same reasoning as in the proof of Proposition 4.4 shows that $x_{i, i+1}=x_{1}^{p^{r-1}}$. For $\ell>2$, one cannot simply argue by weights that $x_{i, j}=0$ for $j>i+1$ so we proceed in a different way.

Since the cohomology class $e_{r} \in H^{2 p^{r-1}}\left(G L_{p}, M_{p}(k)^{(r)}\right)$ is invariant under the action of $G L_{p}$ by conjugation on $G L_{p}$ and $M_{p}(k)^{(r)}$, we conclude that the class $e_{r}\left(\mathbb{G}_{a(\ell)}, V_{\alpha}\right)$ is invariant under the action of $\mathbb{G}_{a}$ (where the action of $\mathbb{G}_{a}$ on $M_{p}(k)$ is the composition of the standard action by conjugation of $G L_{p}$ and the homomorphism $\exp _{\alpha}: \mathbb{G}_{a} \rightarrow G L_{p}$ and the action of $\mathbb{G}_{a}$ on $\mathbb{G}_{a(\ell)}$ is trivial). This remark implies immediately that the matrix

$$
\begin{aligned}
\left(x_{i, j}\right)_{1 \leq i, j \leq p}=e_{r}\left(\mathbb{G}_{a(\ell)}, V_{\alpha}\right) & \in H^{2 p^{r-1}}\left(\mathbb{G}_{a(\ell)}, k\right) \otimes M_{p}(k)^{(r)} \\
& \subset M_{p}\left(H^{e v}\left(\mathbb{G}_{a(\ell)}, k\right)\right)
\end{aligned}
$$

commutes with $\alpha^{(r)}=\alpha$, i.e. $x_{i, j}=x_{i^{\prime}, j^{\prime}}$ whenever $j-i=j^{\prime}-i^{\prime}$. Thus we may rewrite (4.7.1) as follows:

$$
e_{r}\left(\mathbb{G}_{a(\ell)}, V_{\alpha}\right)=\sum_{m=1}^{p-1} y_{m} \otimes\left(\alpha^{(r)}\right)^{m}
$$

where $y_{m} \in H^{2 p^{r-1}}\left(\mathbb{G}_{a(\ell)}, \mathbb{F}_{p}\right)$ has weight $p^{r} m$ and $y_{1}=x_{1}^{p^{r-1}}$. Note that the formula (4.7.2), established above in case $\alpha$ is a $p \times p$ Jordan block, actually holds for any $p$-nilpotent matrix $\alpha$ in view of Lemma 4.3.

Let $\alpha \in M_{n}(k), \beta \in M_{m}(k)$ be a pair of $p$-nilpotent matrices. Proposition 3.8 and Theorem 4.6 give the following formula:

$$
\begin{aligned}
e_{r}\left(\mathbb{G}_{a(\ell)}^{\times 2}, V_{\alpha} \otimes V_{\beta}\right)= & e_{r}\left(\mathbb{G}_{a(\ell)}, V_{\alpha}\right) \otimes 1+1 \otimes e_{r}\left(\mathbb{G}_{a(\ell)}, V_{\beta}\right) \\
& +\sum_{i+j=p^{r-1}, i \neq 0 \neq j} e_{r}(i)\left(\mathbb{G}_{a(\ell)}, V_{\alpha}\right) \otimes e_{r}(j)\left(\mathbb{G}_{a(\ell)}, V_{\beta}\right) .
\end{aligned}
$$

The terms in the above formula corresponding to $i \neq 0 \neq j$ are already known; moreover the corresponding term can be nonzero only if $s(i)<p, s(j)<p$. Finally it's easy to see that if $i+j=p^{r-1}$ and $s(i)<p, s(j)<p$, then $i, j \equiv 0 \bmod p^{r-2}$. This remark, Lemma 4.3 and formula (4.7.2) give

(4.7.3). Let $\alpha, \beta \in M_{n}(k)$ be a pair of commuting $p$-nilpotent matrices. Then the following formula holds:

$$
\begin{aligned}
e_{r}\left(\mathbb{G}_{a(\ell)}^{\times 2}, W_{(\alpha, \beta)}\right)= & \sum_{m=1}^{p-1}\left(y_{m} \otimes 1\right) \otimes\left(\alpha^{(r)}\right)^{m}+\sum_{m=1}^{p-1}\left(1 \otimes y_{m}\right) \otimes\left(\beta^{(r)}\right)^{m} \\
& +\sum_{i+j=p, i \neq 0 \neq j} \frac{x_{2}^{i p^{r-2}} \otimes x_{2}^{j p^{r-2}}}{i ! \cdot j !} \otimes\left(\alpha^{(r)}\right)^{i} \cdot\left(\beta^{(r)}\right)^{j} .
\end{aligned}
$$




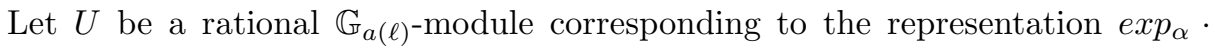
$\exp _{\beta}=\exp _{\alpha+\beta} \cdot \exp _{\xi}^{(1)}($ see (4.6.0)). Proceeding in the same way as in the proof of Theorem 4.6, we get two answers for $e_{r}\left(\mathbb{G}_{a(\ell)}, U\right)$ :

$$
\begin{aligned}
e_{r}\left(\mathbb{G}_{a(\ell)}, U\right)=\sum_{m=1}^{p-1} y_{m} \otimes & \left(\left(\alpha^{(r)}\right)^{m}+\left(\beta^{(r)}\right)^{m}\right)+\sum_{i+j=p, 0 \neq i \neq p} \frac{x_{2}^{p^{r-1}}}{i ! \cdot j !} \otimes\left(\alpha^{(r)}\right)^{i} \cdot\left(\beta^{(r)}\right)^{j}, \\
e_{r}\left(\mathbb{G}_{a(\ell)}, U\right)= & \sum_{m=1}^{p-1} y_{m} \otimes\left((\alpha+\beta)^{(r)}\right)^{m}+\sum_{m=1}^{p-1} F^{*}\left(y_{m}\right) \otimes\left(\xi^{(r)}\right)^{m} \\
& +\sum_{i+j=p, 0 \neq i \neq p} \frac{x_{2}^{i p^{r-2}} \cdot x_{3}^{j p^{r-2}}}{i ! \cdot j !} \otimes\left((\alpha+\beta)^{i} \cdot \xi^{j}\right)^{(r)} .
\end{aligned}
$$

Note that $(\alpha+\beta)^{i} \cdot \xi^{j}$ is a homogeneous polynomial in $\alpha, \beta$ of degree $i+p j=$ $(i+j)+(p-1) j \geq 2 p-1$ and hence equals zero. The same reasoning shows that $\xi^{2}=0$. Thus the last formula simplifies to

$$
e_{r}\left(\mathbb{G}_{a(\ell)}, U\right)=\sum_{m=1}^{p-1} y_{m} \otimes\left((\alpha+\beta)^{(r)}\right)^{m}+x_{2}^{p^{r-1}} \otimes \xi^{(r)} .
$$

Comparing the two answers, we get

$$
\sum_{m=2}^{p-1} y_{m} \otimes\left((\alpha+\beta)^{m}-\alpha^{m}-\beta^{m}\right)^{(r)}=0 .
$$

Taking now $\alpha$ (resp. $\beta$ ) to be multiplication by $x$ (resp. $y$ ) in the $\operatorname{ring} B=$ $k[x, y] /\left(x^{p}, y^{p}\right)$ (in which case the monomials $\alpha^{i} \cdot \beta^{j}$ with $0 \leq i<p, 0 \leq j<p$ are linearly independent over $k$ ) we conclude immediately that $y_{m}=0$ for $m>1$.

Corollary 4.8. Let $\underline{\alpha}=\left(\alpha_{0}, \ldots, \alpha_{\ell-1}\right) \in M_{n}(A)^{\times \ell}$ be an $\ell$-tuple of p-nilpotent, pairwise commuting $n \times n$ matrices over a commutative $\mathbb{F}_{p}$-algebra $A$. Then for any $0 \leq j<p^{r}$

$$
\begin{aligned}
e_{r}(j)\left(\left(\mathbb{G}_{a(r)}^{\times \ell}\right)_{A}, W_{\underline{\alpha}}\right)= & \sum_{\substack{j_{0}+\ldots+j_{\ell-1}=j \\
0 \leq j_{0}, \ldots, j_{\ell-1}}} \frac{x_{r}^{j_{0,0}} \cdot \ldots \cdot x_{1}^{p^{r-1} j_{0, r-1}}}{j_{0,0} ! \cdot \ldots \cdot j_{0, r-1} !} \otimes \ldots \otimes \\
& \otimes \frac{x_{r}^{j_{\ell-1,0}} \cdot \ldots \cdot x_{1}^{p^{r-1} j_{\ell-1, r-1}}}{j_{\ell-1,0} ! \cdot \ldots \cdot j_{\ell-1, r-1} !} \otimes\left(\alpha_{0}^{(r)}\right)^{s\left(j_{0}\right)} \cdot \ldots \cdot\left(\alpha_{\ell-1}^{(r)}\right)^{s\left(j_{\ell-1}\right)}
\end{aligned}
$$

where $j_{i, 0}, \ldots, j_{i, r-1}$ are $p$-adic digits of $j_{i}$ and $s\left(j_{i}\right)=j_{i, 0}+\ldots+j_{i, r-1}$ is their sum.

Proof. Lemma 4.3 shows that it suffices to consider the case when $A=\mathbb{F}_{p}$ and

$$
\alpha_{0}=\alpha \otimes \ldots \otimes 1, \ldots, \alpha_{\ell-1}=1 \otimes \ldots \otimes \alpha
$$

where $\alpha$ is a $p \times p$ Jordan block. In this case, our statement follows from Proposition 3.8, the determination that $\lambda_{i, j}=1$ in Theorem 4.6, and Theorem 4.7.

We now present our explicit computation of the characteristic classes $e_{r}(j)\left(\left(\mathbb{G}_{a(r)}\right)_{A}, V_{\underline{\alpha}}\right)$. 
Theorem 4.9. Let $\underline{\alpha}=\left(\alpha_{0}, \ldots, \alpha_{r-1}\right) \in M_{n}(A)^{\times r}$ be an $r$-tuple of pairwise commuting p-nilpotent matrices over a commutative $\mathbb{F}_{p}$-algebra $A$. Then for any $0 \leq$ $j<p^{r}$

$$
\begin{aligned}
e_{r}(j)\left(\left(\mathbb{G}_{a(r)}\right)_{A}, V_{\underline{\alpha}}\right) & =\sum_{\substack{j_{0}+p j_{1}+\ldots+p^{r-1} j_{r-1}=j \\
0 \leq j_{0}, \ldots, j_{r-1}}} \frac{x_{r}^{j_{0,0}} \cdot \ldots \cdot x_{1}^{p^{r-1} j_{0, r-1}}}{j_{0,0} ! \cdot \ldots \cdot j_{0, r-1} !} \\
& \frac{x_{r}^{p j_{1,0}} \cdot \ldots \cdot x_{2}^{p^{r-1} j_{1, r-2}}}{j_{1,0} ! \cdot \ldots \cdot j_{1, r-2} !} \cdot \ldots \cdot \frac{x_{r}^{p^{r-1} j_{r-1,0}}}{j_{r-1,0} !} \otimes\left(\alpha_{0}^{(r)}\right)^{s\left(j_{0}\right)} \cdot \ldots \cdot\left(\alpha_{r-1}^{(r)}\right)^{s\left(j_{r-1}\right)}
\end{aligned}
$$

where $j_{i, 0}, \ldots, j_{i, r-1-i}$ are $p$-adic digits of $j_{i}$ (note that $j_{i}<p^{r-i}$ ) and $s\left(j_{i}\right)=$ $j_{i, 0}+\ldots+j_{i, r-1-i}$ is their sum.

Proof. The characteristic class $e_{r}(j)\left(\left(\mathbb{G}_{a(r)}\right)_{A}, V_{\underline{\alpha}}\right)$ coincides with the pull-back of $e_{r}(j)\left(\left(\mathbb{G}_{a(r)}\right)_{A}^{\times r}, W_{\underline{\alpha}}\right)$ under the morphism

$$
\mathbb{G}_{a(r)} \stackrel{1 \times F \times \ldots \times F^{r-1}}{\longrightarrow} \mathbb{G}_{a(r)}^{\times r}
$$

Thus, our statement follows from Corollary 4.8 once we observe that the pull-back of the summand corresponding to $j_{0}, \ldots, j_{r-1}$ in (4.8.1) is zero unless $j_{i} \equiv 0 \bmod p^{i}$ for all $i$.

Corollary 4.10. In conditions and notation of Theorem 4.9, the coefficient with which $x_{r}^{j}$ appears in $e_{r}(j)\left(\left(\mathbb{G}_{a(r)}\right)_{A}, V_{\underline{\alpha}}\right)$ is equal to $\frac{\left(\alpha_{0}^{(r)}\right)^{j_{0}} \cdot \ldots \cdot\left(\alpha_{r-1}^{(r)}\right)^{j_{r-1}}}{j_{0} ! \cdot \ldots \cdot j_{r-1} !}$ where $j_{0}, \ldots$, $j_{r-1}$ are $p$-adic digits of $j$. In particular the coefficient with which $x_{r}^{p^{\ell-1}}$ appears in $e_{\ell}^{(r-\ell)}\left(\left(\mathbb{G}_{a(r)}\right)_{A}, V_{\underline{\alpha}}\right)$ is equal to $\alpha_{r-\ell}^{(r)}$.

Proof. We get powers of $x_{r}$ in the formula of Theorem 4.9 only when $0 \leq j_{i}<p$ for all $i$, i.e. when $j_{1}, \ldots, j_{r-1}$ are $p$-adic digits of $j$.

$$
\S 5 \text {. The Morphism } \Theta: V_{r}(G) \rightarrow M_{n}(k)^{\times r}
$$

In Theorem 5.2, we identify the map $\psi: H^{e v}\left(G L_{n(r)}, k\right) \rightarrow k\left[V_{r}\left(G L_{n}\right)\right]$ on the subalgebra of $H^{e v}\left(G L_{n(r)}, k\right)$ determined by the universal classes $e_{\ell}^{(r-\ell)}$. This is done by using the explicit computation of $e_{r}(j)\left(\mathbb{G}_{a(r)}, V_{\underline{\alpha}}\right)$ given in Theorem 4.9 and Corollary 4.10. As we see in several corollaries, this identification gives considerable information concerning the cohomology of infinitesimal group schemes. We then proceed to discuss some plausible refinements of these results.

Let $n, r>0$ be a pair of integers. For each $0 \leq \ell \leq r-1$, the characteristic class $e_{r-\ell}^{(\ell)}\left(G L_{n(r)}, k^{n}\right) \in H^{2 p^{r-\ell-1}}\left(G L_{n(r)}, k\right) \otimes M_{n}(k)^{(r)}$ defines a $k$-linear map $g l_{n}^{(r) \#} \rightarrow$ $H^{2 p^{r-\ell-1}}\left(G L_{n(r)}, k\right)$ and hence a homomorphism of commutative $k$-algebras

$$
S^{*}\left(g l_{n}^{(r) \#}\right) \rightarrow H^{e v}\left(G L_{n(r)}, k\right) .
$$

Taking the product of these homomorphisms over all $\ell(0 \leq \ell \leq r-1)$ we get a homomorphism

$$
\phi: S^{*}\left(\bigoplus_{\ell=0}^{r-1} g l_{n}^{(r) \#}\right) \rightarrow H^{e v}\left(G L_{n(r)}, k\right)
$$


of commutative $k$-algebras and the associated morphism of schemes

$$
\Phi: S p e c H^{e v}\left(G L_{n(r)}, k\right) \rightarrow\left(g l_{n}^{(r)}\right)^{\times r}=g l_{n}^{\times r} .
$$

The main result of $[\mathrm{F}-\mathrm{S}]$ shows that the morphism $\Phi$ is finite. The following proposition is a partial description of its image.

Proposition 5.1. The image of $\Phi:$ Spec $H^{e v}\left(G L_{n(r)}, k\right) \rightarrow g l_{n}^{\times r}$ is contained in $V_{r}\left(G L_{n}\right) \subset g l_{n}^{\times r}$.

Proof. Let $X^{i, j}(\ell)(1 \leq i, j \leq n, 0 \leq \ell \leq r-1)$ denote the coordinate function on $g l_{n}^{\times r}$ which sends $\left(\alpha_{0}, \ldots, \alpha_{r-1}\right) \in M_{n}(k)^{\times r}$ to the $(i, j)$-th entry of $\alpha_{\ell}$. The ideal defining the closed subscheme $V_{r}\left(G L_{n(r)}\right) \subset g l_{n}^{\times r}$ is generated by functions of the form (see Theorem 1.5)

$$
\begin{aligned}
& \sum_{t} X^{i, t}(\ell) \cdot X^{t, j}\left(\ell^{\prime}\right)-X^{i, t}\left(\ell^{\prime}\right) \cdot X^{t, j}(\ell), \\
& \sum_{t_{1}, \ldots, t_{p-1}} X^{i, t_{1}}(\ell) \cdot X^{t_{1}, t_{2}}(\ell) \cdot \ldots \cdot X^{t_{p-1}, j}(\ell) .
\end{aligned}
$$

Thus it suffices to check that $\phi$ takes the above generators to zero. The image of $X^{i, j}(\ell)$ under $\phi$ is equal to the $(i, j)$-th entry of the matrix $e_{r-\ell}^{(\ell)}\left(G L_{n(r)}, k^{n}\right)$. Thus the image under $\phi$ of the first of the above generators is the $(i, j)$-th entry of the matrix

$$
e_{r-\ell}^{(\ell)}\left(G L_{n(r)}, k^{n}\right) \cdot e_{r-\ell^{\prime}}^{\left(\ell^{\prime}\right)}\left(G L_{n(r)}, k^{n}\right)-e_{r-\ell^{\prime}}^{\left(\ell^{\prime}\right)}\left(G L_{n(r)}, k^{n}\right) \cdot e_{r-\ell}^{(\ell)}\left(G L_{n(r)}, k^{n}\right)
$$

whereas the image under $\phi$ of the second generator is the $(i, j)$-th entry of the matrix $\left(e_{r-\ell}^{(\ell)}\left(G L_{n(r)}, k^{n}\right)\right)^{p}$. But the above matrices are zero according to Theorem 3.1 .

Proposition 5.1 shows that the homomorphism $\phi$ defines a homomorphism of commutative $k$-algebras

$$
\bar{\phi}: k\left[V_{r}\left(G L_{n}\right)\right] \rightarrow H^{e v}\left(G L_{n(r)}, k\right) .
$$

Let $\Phi, \Psi$ denote the maps of affine schemes associated to the maps $\bar{\phi}, \psi$ of commutative $k$-algebras.

Theorem 5.2. The composition

$$
\Theta: V_{r}\left(G L_{n(r)}\right) \stackrel{\Psi}{\longrightarrow} \operatorname{Spec} H^{e v}\left(G L_{n(r)}, k\right) \stackrel{\Phi}{\longrightarrow} V_{r}\left(G L_{n(r)}\right)
$$

equals the $r$-th Frobenius twist morphism corresponding to the scheme $V_{r}\left(G L_{n(r)}\right)=$ $V_{r}\left(G L_{n}\right)$ (defined over $\left.\mathbb{F}_{p}\right)$.

Proof. It suffices to show that for $k=\mathbb{F}_{p}$ the ring homomorphism

$$
\theta: \mathbb{F}_{p}\left[V_{r}\left(G L_{n(r)}\right)\right] \stackrel{\bar{\phi}}{\longrightarrow} H^{e v}\left(G L_{n(r)}, \mathbb{F}_{p}\right) \stackrel{\psi}{\longrightarrow} \mathbb{F}_{p}\left[V_{r}\left(G L_{n(r)}\right)\right]
$$

coincides with the $p^{r}$-th power map. Denote the $\mathbb{F}_{p}$-algebra $\mathbb{F}_{p}\left[V_{r}\left(G L_{n(r)}\right)\right]$ by $A$. The universal group homomorphism

$$
\exp _{\underline{\alpha}}: \mathbb{G}_{a(r)} \otimes_{\mathbb{F}_{p}} A \rightarrow G L_{n(r)} \otimes_{\mathbb{F}_{p}} A
$$

is determined by the universal $r$-tuple of pairwise commuting $p$-nilpotent matrices $\underline{\alpha}=\left(\alpha_{0}, \ldots, \alpha_{r-1}\right) \in M_{n}(A)^{\times r}$, where $\alpha_{\ell}=\left(X^{i, j}(\ell)\right)_{i, j=1}^{n}$. The definition of $\phi$ implies that $\phi\left(X^{i, j}(\ell)\right)$ coincides with the $(i, j)$-th entry of the matrix 
$e_{r-\ell}^{(\ell)}\left(G L_{n(r)}, \mathbb{F}_{p}^{n}\right)$. The naturality of the classes $e_{r}$ implies that the map $u^{*}$ of the proof of Theorem 1.14 sends this coefficient to the corresponding coefficient of the matrix $e_{r-\ell}^{(\ell)}\left(\mathbb{G}_{a(r)}, V_{\underline{\alpha}}\right)$. Hence, $\psi \phi\left(X^{i, j}(\ell)\right)$ coincides with the coefficient of $x_{r}^{p^{r-\ell-1}}$ in the $(i, j)$-th entry of the matrix $e_{r-\ell}^{(\ell)}\left(\mathbb{G}_{a(r)}, V_{\underline{\alpha}}\right)$. Corollary 4.10 shows that this coefficient equals $X^{i, j}(\ell)^{p^{r}}$.

As we make explicit in the following (immediate) corollary of Theorem 5.2, the theorem provides a reasonably explicit subalgebra of $H^{e v}\left(G L_{n(r)}, k\right)$.

Corollary 5.3. If $f \in \operatorname{Ker} \bar{\phi}$, then $f^{p^{r}}=0$.

Remark 5.4. Consider the special case $r=1$. The scheme

$$
V_{1}\left(G L_{n}\right)=N_{p}\left(g l_{n}\right) \subset g l_{n}=M_{n}(k)
$$

is defined by setting all entries of the matrix $\alpha^{p}$ (where $\alpha=\left(X^{i, j}\right)_{i, j=1}^{n}$ ) to be zero. Since the trace of $\alpha$ lies in $\operatorname{Ker} \bar{\phi}$ ([F-S], 1.4), this kernel is always nontrivial. According to a theorem of Friedlander and Parshall [F-P] (with bound on $p$ improved in $[\mathrm{A}-\mathrm{J}])$, for $p>n$ the homomorphism

$$
\phi: S^{*}\left(M_{n}(k)^{\#}\right)=k\left[X^{i, j}\right]_{i, j=1}^{n} \rightarrow H^{e v}\left(G L_{n(1)}, k\right)
$$

is surjective and its kernel is precisely the ideal generated by the coefficients of the characteristic polynomial of $\alpha$. Apparently the same is true for $n=p$. However for $p<n$ the situation appears more complicated as can be seen in examples computed in $[\mathrm{A}-\mathrm{J}]$.

Corollary 5.5. Let $G / k$ be an infinitesimal group scheme of height $\leq r$. The image of the canonical homomorphism

$$
\psi: H^{e v}(G, k) \rightarrow k\left[V_{r}(G)\right]
$$

contains $k\left[V_{r}(G)\right]^{p^{r}}$.

Proof. Choose a closed embedding $G \hookrightarrow G L_{n(r)}$. Since the homomorphism $k\left[V_{r}\left(G L_{n(r)}\right)\right] \rightarrow k\left[V_{r}(G)\right]$ is surjective, the naturality of $\psi$ given by Theorem 1.14 implies that it suffices to consider the case $G=G L_{n(r)}$. In this case, our statement follows immediately from Theorem 5.2.

The following question is motivated by Theorem 5.2 and the verification in Theorem 5.11 of one special case.

Question 5.6. With notation as in Theorem 5.2, does the composition

$$
\text { Spec } H^{e v}\left(G L_{n(r)}, k\right) \stackrel{\Phi}{\longrightarrow} V_{r}\left(G L_{n(r)}\right) \stackrel{\Psi}{\longrightarrow} \operatorname{Spec} H^{e v}\left(G L_{n(r)}, k\right)
$$

also equal the $r$-th Frobenius twist morphism?

An affirmative answer to this question would imply in particular that $z^{p^{r}}=0$ for any element $z \in \operatorname{Ker}\left(\psi: H^{e v}\left(G L_{n(r)}, k\right) \rightarrow k\left[V_{r}\left(G L_{n(r)}\right)\right]\right)$. We show in [SFB] that each element in $\operatorname{Ker} \psi$ is nilpotent, which gives additional evidence in favor of an affirmative answer.

Question 5.7. Let $G / k$ be an infinitesimal group scheme of height $\leq r$. The choice of a closed embedding $i: G \hookrightarrow G L_{n(r)}$ defines a morphism of schemes

$$
\Phi_{G}: \operatorname{Spec} H^{e v}(G, k) \stackrel{i_{*}}{\longrightarrow} \operatorname{Spec} H^{e v}\left(G L_{n(r)}, k\right) \stackrel{\Phi}{\longrightarrow} V_{r}\left(G L_{n(r)}\right)=V_{r}\left(G L_{n(r)}\right)^{(r)}
$$


Is it the case that $\Phi_{G}\left(\operatorname{Spec} H^{e v}(G, k)\right) \subset V_{r}(G)^{(r)}$ and that the resulting morphism $\Phi_{G}:$ Spec $H^{e v}(G, k) \rightarrow V_{r}(G)^{(r)}$ is independent of the choice of the embedding $G \hookrightarrow G L_{n(r)} ?$

Let $G$ be a group scheme of height 1 with Lie algebra $g$. Then the edge homomorphism in the May spectral sequence for $H^{*}(G, k)$,

$$
S^{*}\left(g^{\#(1)}\right)=E_{2}^{2 *, 0} \rightarrow E_{\infty}^{2 *, 0} \subset H^{e v}(G, k),
$$

determines a map

$$
\text { Spec } H^{e v}(G, k) \rightarrow g^{(1)} .
$$

The naturality of the May spectral sequence implies that if $G$ is provided with a closed embedding $G \subset G L_{n(1)}$, then the composition of (5.8.0) and the map $g^{(1)} \rightarrow g l_{n}^{(1)}$ induced by this closed embedding equals the map $\Phi_{G}$ of Question 5.7.

Thus, the following corollary of Proposition 5.1 (first obtained by J. Jantzen [J1] by other means) gives an affirmative answer to Question 5.7 in the special case $r=1$.

Corollary 5.8. Let $G$ be a group scheme of height 1 with Lie algebra $g$. Then the morphism (5.8.0) has image contained in $V_{1}(G)^{(1)}=N_{p}(g)^{(1)} \subset g^{(1)}$.

Proof. By Proposition 5.1 and the naturality of the May spectral sequence, it suffices to verify that the scheme-theoretic intersection of $N_{p}\left(g l_{n}\right)$ and $g$ equals $N_{p}(g)$. This follows by comparing $A$-valued points of $N_{p}\left(g l_{n}\right) \cap g$ and $N_{p}(g)$ for any commutative $k$-algebra $A$.

Remark 5.9. If the answer to Question 5.7 is affirmative in general, then Theorem 5.2 admits the following generalization:

For any infinitesimal group scheme $G / k$ of height $\leq r$, the composition

$$
V_{r}(G) \stackrel{\Psi}{\longrightarrow} \operatorname{Spec} H^{e v}(G, k) \stackrel{\Phi_{G}}{\longrightarrow} V_{r}(G)^{(r)}
$$

equals the $r$-th Frobenius twist morphism.

Question 5.10. If the answer to Question 5.7 is affirmative, then one can go further and ask the following: for any infinitesimal group scheme $G / k$ of height $\leq r$, does the composition

$$
\text { Spec } H^{\text {ev }}(G, k) \stackrel{\Phi_{G}}{\longrightarrow} V_{r}(G)^{(r)} \stackrel{\Psi^{(r)}}{\longrightarrow}\left(\operatorname{Spec} H^{e v}(G, k)\right)^{(r)}
$$

equal the r-th Frobenius twist morphism?

The following theorem provides an affirmative answer to Question 5.10 in the very special case for which $r=1, p=2$.

Theorem 5.11. Let $G$ be an infinitesimal group scheme of height $\leq 1$ over a field $k$ of characteristic 2 . Then the composition

$$
\text { Spec } H^{*}(G, k) \stackrel{\Phi_{G}}{\longrightarrow} V_{1}(G) \stackrel{(1)}{\stackrel{\Psi^{(1)}}{\longrightarrow}}\left(\operatorname{Spec} H^{*}(G, k)\right)^{(1)}
$$

equals the Frobenius twist morphism.

Proof. The proof is based on the use of the following invariant version of May's resolution $[\mathrm{M}]$. Let $g$ be a finite-dimensional restricted Lie algebra over a field $k$ of 
characteristic 2. Denote by $U=U(g)$ the universal restricted enveloping algebra of $g$. Consider the complex

$$
0 \leftarrow k \stackrel{\epsilon}{\longleftarrow} U \stackrel{d}{\longleftarrow} U \otimes g \stackrel{d}{\longleftarrow} U \otimes \Gamma^{2}(g) \stackrel{d}{\longleftarrow} \cdots .
$$

The differential $d: U \otimes \Gamma^{n}(g) \rightarrow U \otimes \Gamma^{n-1}(g)$ is a sum of two maps. The first one is the $U$-linear extension of the canonical embedding

$$
\Gamma^{n}(g) \hookrightarrow g \otimes \Gamma^{n-1}(g) \hookrightarrow U \otimes \Gamma^{n-1}(g) .
$$

The second is defined as follows. The squaring map $g \rightarrow g\left(x \mapsto x^{[2]}\right)$ is a homogeneous polynomial of degree 2 and hence defines a $k$-linear map $\xi: \Gamma^{2}(g) \rightarrow g$. Consider the $k$-linear homomorphism

$$
\begin{aligned}
\xi_{n}: \Gamma^{n}(g) \stackrel{\text { comult }}{\longrightarrow} & \bigoplus_{i=0}^{n-2} \Gamma^{i}(g) \otimes \Gamma^{2}(g) \otimes \Gamma^{n-i-2}(g) \\
& \stackrel{\oplus_{i} 1 \otimes \xi \otimes 1}{\longrightarrow} \bigoplus_{i=0}^{n-2} \Gamma^{i}(g) \otimes g \otimes \Gamma^{n-i-2}(g) \stackrel{\text { can }}{\longrightarrow} g^{\otimes(n-1)} .
\end{aligned}
$$

Extending scalars if necessary, we may assume that the field $k$ is infinite. In this case the vector space $\Gamma^{n}(g)$ is generated by tensors of the form $x^{\otimes n}(x \in g)$. Since $\xi_{n}\left(x^{\otimes n}\right)=x^{[2]} \otimes \ldots \otimes x+\ldots+x \otimes \ldots \otimes x^{[2]} \in \Gamma^{n-1}(g)$, we conclude that $\xi_{n}$ is a $k$-linear homomorphism from $\Gamma^{n}(g)$ to $\Gamma^{n-1}(g)$. Now the second summand in $d$ is defined to be the $U$-linear extension of $\xi_{n}$. A straightforward verification shows that $d$ is really a differential; moreover using the results of May $[\mathrm{M}]$ or arguing directly one shows that (5.11.1) is a $U$-projective resolution of $k$. We may use this resolution to compute the (co)homology of $g$. Thus we see in particular that $H^{*}(G, k)$ coincides with homology of the complex (cf. [F-P])

$$
0 \rightarrow g^{\#} \stackrel{\xi^{\#}}{\longrightarrow} S^{2}\left(g^{\#}\right) \stackrel{\xi_{3}^{\#}}{\longrightarrow} S^{3}\left(g^{\#}\right) \rightarrow \cdots .
$$

The complex (5.11.2) is a commutative differential graded $k$-algebra and multiplication in cohomology is induced by multiplication in this complex.

Denote the coordinate algebra $k\left[V_{1}(G)\right]=k\left[N_{p}(g)\right]$ by $A$. Thus $A$ is a factor algebra of $S^{*}\left(g^{\#}\right)$ modulo the ideal generated by $\xi^{\#}\left(g^{\#}\right) \subset S^{2}\left(g^{\#}\right)$. The universal homomorphism $g_{a} \otimes A \rightarrow g \otimes A$ is defined by the element $1_{g} \in g \otimes g^{\#} \subset g \otimes A$. The dual map $g^{\#} \otimes A \rightarrow g_{a}^{\#} \otimes A=A$ is an $A$-linear extension of the canonical embedding $\lambda: g^{\#} \hookrightarrow A$. Our definitions imply that the homomorphism $\psi: H^{*}(G, k) \rightarrow A$ is given by the following homomorphism of complexes:

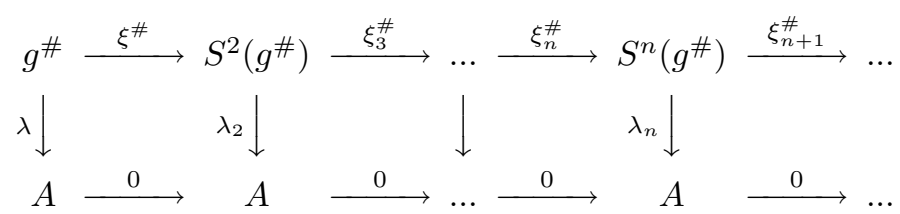

where $\lambda_{n}: S^{n}\left(g^{\#}\right) \rightarrow A$ is the $n$-th component of the canonical projection $S^{*}\left(g^{\#}\right) \rightarrow$ $A$. In other words, the canonical projection $S^{*}\left(g^{\#}\right) \rightarrow A$ is a homomorphism of differential graded algebras ( $A$ is equipped with zero differential) and the induced map in cohomology is our homomorphism $\psi$. On the other hand, the homomorphism $\phi_{G}: A^{(1)} \rightarrow H^{*}(G, k)$ is induced by the homomorphism $S^{*}\left(g^{\#(1)}\right) \rightarrow H^{*}(G, k)$ arising from the $k$-linear map $g^{\#(1)} \hookrightarrow S^{2}\left(g^{\#}\right): x^{(1)} \mapsto x^{2}$. A straightforward computation completes the proof. 


\section{§6. An APPliCATION TO DISCRETE GROUP HOMOLOGY}

In [F-S], information about the rational cohomology groups $H^{*}\left(G L_{n}, g l_{n}^{(r)}\right)$ was used to compute homology of the discrete group $G L\left(\mathbb{F}_{q}\right)$ with coefficients in the adjoint representation. In this section, we show that our present knowledge of comultiplication in $H^{*}\left(G L_{n}, g l_{n}^{(r)}\right)$ enables us to determine the multiplicative structure of $H_{*}\left(G L\left(\mathbb{F}_{q}\right), M\left(\mathbb{F}_{q}\right)\right)$.

For a field $k$, we denote by $G L_{n}(k)$ the discrete group of invertible $n \times n$ matrices over $k$. The group $G L_{n}(k)$ acts by conjugation on $M_{n}(k)$. We embed $G L_{n}(k)$ into $G L_{n+1}(k)$ (and $M_{n}(k)$ into $M_{n+1}(k)$ ) in a standard way and we set $G L(k)=$ $\lim _{\rightarrow} G L_{n}(k), \quad M(k)=\lim _{\rightarrow} M_{n}(k)$. Since group homology commutes with direct $\overrightarrow{\text { limits, we conclude that }}$

$$
H_{i}(G L(k), M(k))=\lim _{\rightarrow} H_{i}\left(G L_{n}(k), M_{n}(k)\right) .
$$

By a stability theorem of W. Dwyer [D], $H_{i}(G L(k), M(k))=H_{i}\left(G L_{n}(k), M_{n}(k)\right)$ for all $n$ big enough (depending on $i$ ).

Choosing an ordering for the basis of $k^{n} \otimes k^{m}$, we get a homomorphism in the category of pairs (group, module)

$$
\left(G L_{n}(k) \times G L_{m}(k), M_{n}(k) \otimes M_{m}(k)\right) \rightarrow\left(G L_{n m}(k), M_{n m}(k)\right)
$$

which induces a homomorphism in homology

$$
\begin{aligned}
H_{*}\left(G L_{n}(k), M_{n}(k)\right) \otimes & H_{*}\left(G L_{m}(k), M_{m}(k)\right) \\
=H_{*}\left(G L_{n}(k) \times G L_{m}(k), M_{n}(k) \otimes M_{m}(k)\right) & \\
& \rightarrow H_{*}\left(G L_{n m}(k), M_{n m}(k)\right) \rightarrow H_{*}(G L(k), M(k)) .
\end{aligned}
$$

This homomorphism is compatible with stabilization, thereby inducing a pairing

$$
H_{*}(G L(k), M(k)) \otimes H_{*}(G L(k), M(k)) \rightarrow H_{*}(G L(k), M(k))
$$

which makes $H_{*}(G L(k), M(k))$ into an associative graded $k$-algebra. We proceed to determine this algebra in case $k=\mathbb{F}_{q}$ is a finite field.

For an affine group scheme $G / k$ and a rational $G$-module $M$, viewing a regular morphism as a set map on $k$-valued points determines a natural homomorphism $H^{*}(G, M) \rightarrow H^{*}(G(k), M)$. When $G$ and $M$ are defined over $\mathbb{F}_{p}$, the Frobenius twist $M^{(r)}$ may be identified as a $G$-module with $M$ endowed with a new action of $G$ via the $r$-th power of the Frobenius endomorphism $F: G \rightarrow G$. Assume now that $k$ is a finite field. Then the action of $F$ on the group of rational points $G(k)$ is an isomorphism and hence $H^{*}\left(G(k), M^{(r)}\right)=H^{*}(G(k), M)$. Thus, for any $r \geq 0$, we get a homomorphism $H^{*}\left(G, M^{(r)}\right) \rightarrow H^{*}\left(G(k), M^{(r)}\right)=H^{*}(G(k), M)$ and each of the diagrams

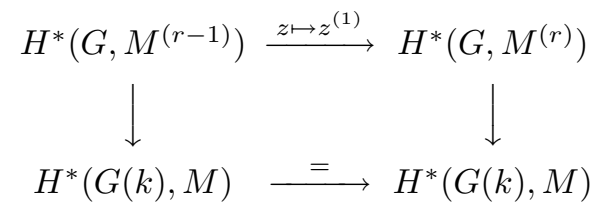

commute. Applying the previous construction to $G=G L_{n}$ and $M=M_{n}(k)$, we obtain canonical homomorphisms (cf. [CPSvdK])

$$
H^{*}\left(G L_{n}, M_{n}(k)^{(r)}\right) \rightarrow H^{*}\left(G L_{n}(k), M_{n}(k)\right) .
$$


Let $\ell \geq 0$ be an integer. Choose $r>0$ such that $\ell<p^{r}$ and denote by $e(\ell)$ the image of $e_{r}(\ell)\left(G L_{n}, k^{n}\right) \in H^{2 \ell}\left(G L_{n}, M_{n}(k)^{(r)}\right)$ in $H^{2 \ell}\left(G L_{n}(k), M_{n}(k)\right)$ $=H_{o m}\left(H_{2 \ell}\left(G L_{n}(k), M_{n}(k)\right), k\right)$. Commutativity of the diagram (6.0) implies that the resulting cohomology class is independent of the choice of $r$. Moreover one checks easily that the diagram

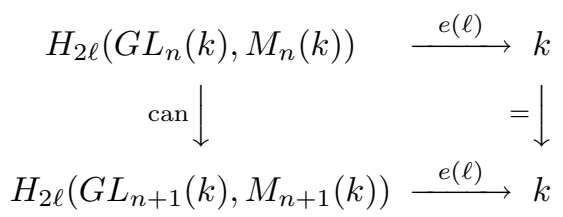

commutes. Passing to a limit in $n$ we get a homomorphism

$$
e(\ell): H_{2 \ell}(G L(k), M(k)) \rightarrow k .
$$

The following theorem recalls the determination of the additive structure of $H_{*}(G L(k), M(k))$ as presented in [F-S].

Theorem 6.1 ([F-S], Theorem 7.6). Let $k=\mathbb{F}_{q}$ be a finite field. Then

$$
H_{m}(G L(k), M(k))=0 \text { if } m \text { is odd. }
$$

On the other hand, if $m=2 \ell$ is even, then the homomorphism

$$
e(\ell): H_{m}(G L(k), M(k)) \rightarrow k
$$

is an isomorphism.

Denote by $x(\ell) \in H_{2 \ell}(G L(k), M(k))$ the element for which $e(\ell)(x(\ell))=1$. The multiplicative structure of $H_{*}(G L(k), M(k))$ is given by the following theorem.

Theorem 6.2. Let $k=\mathbb{F}_{q}$ be a finite field. Then $x(\ell) \cdot x\left(\ell^{\prime}\right)=x\left(\ell+\ell^{\prime}\right)$. In other words, $H_{*}(G L(k), M(k))$ is a polynomial algebra over $k$ in one generator $x(1) \in H_{2}(G L(k), M(k))$.

Proof. Proposition 3.8 and Theorem 4.6 show that (for $r$ big enough)

$$
e_{r}\left(\ell+\ell^{\prime}\right)\left(G L_{n} \times G L_{n^{\prime}}, k^{n} \otimes k^{n^{\prime}}\right)=\sum_{i+j=\ell+\ell^{\prime}} e_{r}(i)\left(G L_{n}, k^{n}\right) \otimes e_{r}(j)\left(G L_{n^{\prime}}, k^{n^{\prime}}\right) .
$$

This formula implies the commutativity of the following diagram:

$$
\begin{aligned}
& H_{2 \ell}\left(G L_{n}(k), M_{n}(k)\right) \otimes H_{2 \ell^{\prime}}\left(G L_{n^{\prime}}(k), M_{n^{\prime}}(k)\right) \stackrel{e(\ell) \otimes e\left(\ell^{\prime}\right)}{\longrightarrow} k \\
& H_{2\left(\ell+\ell^{\prime}\right)}\left(G L_{n n^{\prime}}(k), M_{n n^{\prime}}(k)\right) \stackrel{e\left(\ell+\ell^{\prime}\right)}{\longrightarrow} k .
\end{aligned}
$$

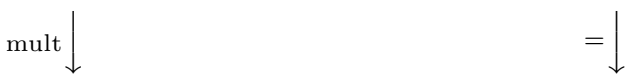

Now we conclude immediately that $e\left(\ell+\ell^{\prime}\right)\left(x(\ell) x\left(\ell^{\prime}\right)\right)=e(\ell)(x(\ell)) \cdot e\left(\ell^{\prime}\right)\left(x\left(\ell^{\prime}\right)\right)=1$, i.e. $x(\ell) x\left(\ell^{\prime}\right)=x\left(\ell+\ell^{\prime}\right)$.

\section{REFERENCES}

[A-J] H. Andersen and J. Jantzen, Cohomology of induced representations for algebraic groups, Math. Annalen 269 (1985), 487-525. MR 86g:20057

[CPSvdK] E. Cline, B. Parshall, L. Scott, and W. van der Kallen, Rational and generic cohomology, Inventiones Math. 39 (1977), 143-163. MR 55:12737

[D] W. Dwyer, Twisted homological stability for general linear groups, Annals of Math. 111 (1980), 239-251. MR 81b:18006 
[D-G] M. Demazure and P. Gabriel, Groupes algébriques I (Masson/North-Holland, ed.), 1970. MR 46:1800

[D-M] B. Dundas and R. McCarthy, Stable K-theory and Hochschild homology, Annals of Math. 142 (1994), 685-701. MR 96e:19005a; MR 96e:19005b

[F-P] E. Friedlander and B. Parshall, Cohomology of Lie algebras and algebraic groups, Amer. J. Math. 108 (1986), 235-253. MR 87c:20080

[F-S] E. Friedlander and A. Suslin, Cohomology of finite group schemes over a field, Inventiones Math. 127 (1997), 209-270. CMP 97:06

[G] D. Gross, Higher nullcones and commuting varieties, Comm. in Algebra 21 (1993), 1427-1455. MR 94g:20059

[J1] J. Jantzen, Kohomologie von p-Lie-Algebren und nilpotente Elemente, Abh. Math. Sem. Univ. Hamburg 56 (1986), 191-219. MR 88e:17019

[J] J. Jantzen, Representations of Algebraic Groups (Academic Press, ed.), 1987. MR 89c:20001

[M] J.P. May, The cohomology of restricted Lie algebras and of Hopf algebras, J. Algebra 3 (1966), 123-146. MR 33:1347

[Q] D. Quillen, Higher algebraic K-theory I, Algebraic K-Theory I (Springer-Verlag, Lecture Notes in Math 341, ed.), 1973, pp. 85-147. MR 49:2895

[SFB] A. Suslin, E. Friedlander, and C. Bendel, Support varieties for infinitesimal group schemes, Journal of the American Mathematical Society (this volume).

Department of Mathematics, Northwestern University, Evanston, Illinois 60208

E-mail address: suslin@math.nwu.edu

E-mail address: eric@math.nwu.edu

E-mail address: bendel@math.nwu.edu 